\title{
EFFECTS OF MEDIA SENSATIONALIZATION ON COGNITIVE PERFORMANCE AND POST CONCUSSIVE SYMPTOMS
}

\author{
by \\ CARA ANN BUSSELL
}

B.A., Lewis and Clark College, 2005

\author{
A thesis submitted to the Graduate Faculty of the \\ University of Colorado Colorado Springs \\ in partial fulfillment of the \\ requirements for the degree of \\ Master of Arts \\ Department of Psychology
}

2017 
This thesis for the Master of Arts degree by

\author{
Cara Ann Bussell \\ has been approved for the \\ Department of Psychology
}

by

Brandon E. Gavett, Chair

Thomas A. Pyszczynski

Elizabeth Daniels

Date $\underline{09 / 12 / 2017}$ 
Bussell, Cara Ann (M.A., Psychology)

Effects of Media Sensationalization on Cognitive Performance and Post Concussive Symptoms

Thesis directed by Assistant Professor Brandon E. Gavett

\begin{abstract}
The current study aimed to examine if media about mild traumatic brain injury (mTBI) framed in a sensationalized manner had a negative impact on cognitive functioning (working memory and attention) and persistent mTBI symptoms. Onehundred and two participants $\left(M_{\text {Age }}=37.15 ; \mathrm{SD}=22.61\right)$ with a history of post-acute mTBI, recruited through a community research registry and an undergraduate recruitment system, were included in this study. Participants were assessed with a measure of health literacy, the Short Test of Functional Health Literacy in Adults (S-TOFHLA), and randomized to watch either a sensationalized or non-sensationalized news clip focused on mTBI. They were then assessed with the Paced Auditory Serial Addition Test (PASAT), Rivermead Post Concussion Symptoms Questionnaire (RPQ), Patient Reported Outcome Measures Information System (PROMIS) Depression scale, and the posttraumatic stress disorder (PTSD) Checklist (PCL-5). Bayesian analyses indicated that sensationalized media was not a strong predictor of PASAT score $(\beta=0.04)$ or post-concussion syndrome symptom severity $(\beta=-0.03)$. S-TOFHLA scores and age category were shown to be the best predictors of working memory and attention. Depression symptoms were the best predictor of PCS symptom reporting. Future studies with a larger sample size can improve the precision of these estimated effects.
\end{abstract}




\section{TABLE OF CONTENTS}

\section{CHAPTER}

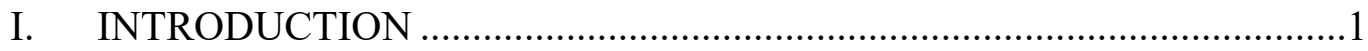

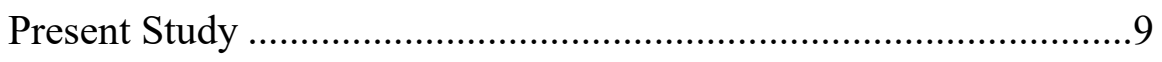

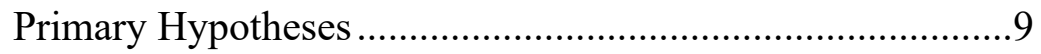

Secondary Hypotheses ..........................................................10

II. METHOD ……………………………………..............................11

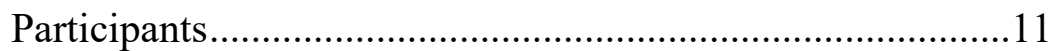

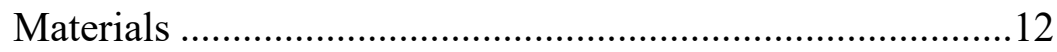

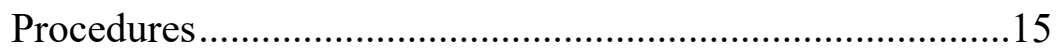

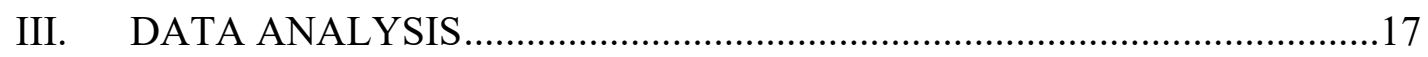

Power Analysis ..................................................................17

Traditional Analysis.............................................................18

Bayesian Analysis ..............................................................19

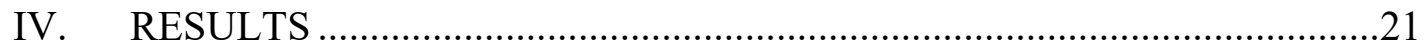

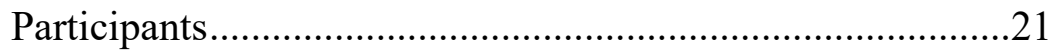

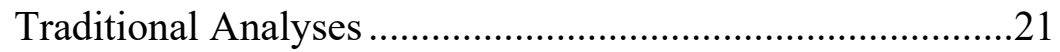

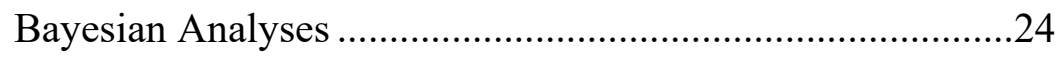

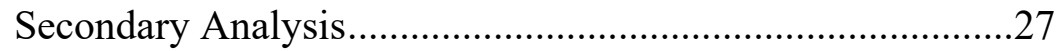

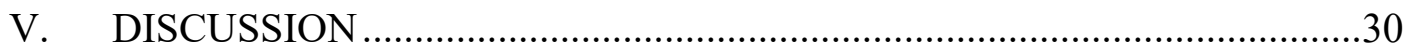

Limitations and Future Research .........................................36 
Conclusion

REFERENCES . 38

APPENDIX A: REVISED HOFFMAN MEASUREMENT. .48

APPENDIX B: IRB APPROVAL . 51 


\section{LIST OF TABLES}

\section{TABLE}

1. Prior Distributions for Predictors used in Bayesian Analyses .......................20

2. Participant Demographics .....................................................................21

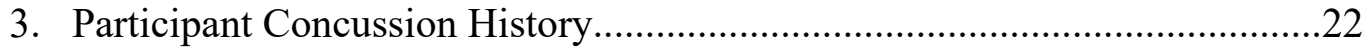

4. Interaction Effects for PASAT Traditional Regression ...............................23

5. Interaction Effects for RPQ Traditional Regression .................................23

6. Interaction Effects for PASAT Bayesian Regression ..................................26

7. Interaction Effects for RPQ Bayesian Regression ....................................27

8. Secondary Interaction Effects for PASAT Bayesian Regression .................28

9. Secondary Interaction Effects for RPQ Bayesian Regression .....................29 


\section{LIST OF FIGURES}

\section{FIGURE}

1. Plot of prior and posterior distributions of parameter estimates for the effect of sensationalized media on PASAT performance ............................25

2. Plot of prior and posterior distributions of parameter estimates for the effect of sensationalized media on RPQ score. 


\section{CHAPTER I}

\section{INTRODUCTION}

The Center for Disease Control and Prevention estimates that 1.7 million people in the United States sustain a traumatic brain injury (TBI) annually (Faul, Xu, Wald, \& Coronado, 2010). The lifetime prevalence of TBI is roughly $12 \%$ (Frost, Farrer, Primosch, \& Hedges, 2013). Approximately 5.3 million Americans are presently living with at least one chronic disability due to TBI (Langlois, Rutland-Brown, \& Wald, 2006). It is estimated that direct medical costs and indirect costs such as lost productivity as a result of these injuries total $\$ 60$ billion annually (Finkelstein, Corso, Miller, 2006).

Three age groups have been shown to be at highest risk for TBI. These include children between the ages of 0 to 4 , adolescents aged 15 to 19 , and adults aged 65 and older (American Psychiatric Association, 2013; Faul et al., 2010). Overall, the most common cause of TBI is falling (American Psychiatric Association, 2013). The highest rates of TBI-related hospitalization and death occur in adults over the age of 75 (Faul et al., 2010). There are several age-related factors that put older adults at risk for a TBI. For example, older adults are at a higher likelihood to have a medical condition such as degenerative joint disease or postural hypotension, which predisposes them to falls and motor vehicle accidents (Ferrell \& Tanev, 2002). Additionally, this age group is also more likely than their younger counterparts to be on medications such as anti-coagulants, which can also increase the risk for falling (Thompson, McCormick, \& Kagan, 2006). 
About $70-90 \%$ of treated head injuries are concussions, which are also known as mild traumatic brain injuries (mTBIs) (Cassidy et al., 2004). The mechanical impact causing the mTBI can cause the axons to be stretched and damaged in a process known as diffuse axonal injury (Smith, Meaney, \& Shull, 2003). This damage initiates a neurometabolic cascade, including ionic fluxes, metabolic abnormalities, and reduced cerebral blood flow (Giza \& Hovda, 2001). In mTBI, this cascade can cause axonal swelling and as a result, a small number of cells may become disconnected and die (Giza \& Hovda, 2001; Iverson, 2005). In general, mTBI patients recover from these neurophysiological effects within 10 days (Giza \& Hovda, 2001). Variables used to diagnose mTBI clinically include a traumatically induced loss of consciousness (LOC) for no longer than 30 minutes, posttraumatic amnesia (PTA) for less than 24 hours and a Glasgow Coma Scale score of 13-15 (American Psychiatric Association, 2013). Uncomplicated mTBIs, as opposed to complicated mTBIs, are generally defined as being on the mild end of these scales, and do not cause any structural lesions to brain tissue (Iverson, 2005). It is common for mTBI patients to suffer from deficits in working memory (Kumar, Rao, Chandramouli, \& Pillai, 2013), attention, and concentration (Paniak et al., 2002). Other signs and symptoms of mTBI include headaches, dizziness, fatigue, irritability, sleep problems, memory deficits, and problems tolerating stress (Reuben, Sampson, Harris, Williams, \& Yates, 2014). The majority of mTBI patients fully recover from the incident within three months (American Psychiatric Association, 2013; Carroll et al., 2004; Cassidy, Boyle, \& Carroll, 2014). Those who display these signs and symptoms after three months are said to suffer from symptoms that are collectively known as post-concussive syndrome (PCS) (Bigler, 2008). 
There is variability among individual studies reporting the percentage of mTBI patients who go on to develop PCS. For example, initial studies estimated this value to be around $15 \%$, but there are also a number of studies with estimated values between up to 43\% (Lannsjö, Geijerstam, Johansson, Bring, \& Borg, 2009; Rohling, Larrabee, \& Millis, 2012; Schneiderman, Braver, \& Kang, 2008; Stålnacke, Björnstig, Karlsson, \& Sojka, 2005). However, there are also a number of studies, including meta-analyses, that support the notion that PCS is essentially absent in population based samples at or beyond 90 days post injury (Frencham, Fox, \& Mayberry, 2005; Rohling et al., 2011). A number of factors have been hypothesized to be responsible for this variability.

McCrea (2008) explained that some earlier studies did not use the same definition of mTBI that is used today. Nor did many of these studies differentiate complicated from uncomplicated mTBI or record the total number of mTBIs the participants had sustained. Also, Belanger et al. (2005) has shown that results differ depending on the type of participants sampled. That is, samples from clinical populations or those involved in litigation tend to show stable or worsening PCS over time, while population based samples tend to achieve full recovery.

Silverberg and Iverson (2011) conducted a thorough review of the literature focused on the etiology of PCS and noted that pre-existing psychological disorders such as anxiety, depression, and traumatic stress have all been shown to contribute to the development of PCS. Iatrogenic risks such as over testing (Roth \& Spencer, 2013) or the recommendation to delay activity for an inadvertently detrimental period of time (DiFazio, Silverberg, Kirkwood, Bernier, \& Iverson, 2015) have also been shown to negatively affect the chance for full recovery. Expectations for recovery and symptom 
misattribution (Snell, Surgenor, Hay-Smith, Williman, \& Siegert, 2015) can be predictive of PCS. Additionally, Silverberg and Iverson (2011) explained that mood and anxiety disorders can also cause many of the same symptoms as PCS, making it difficult to differentiate PCS symptoms from symptoms of the premorbid psychological disorder. Finally, they also acknowledged the impact that phenomena such as stereotype threat, diagnosis threat, and the nocebo effect can have on PCS (Silverberg \& Iverson; 2011).

Steele (1997) described stereotype threat as the impact that allowing negative stereotypes to become self-relevant can have on abilities such as cognitive functioning. Kit, Manteer, Tuokko, and Spencer-Rogers (2014) showed that some cognitive performance measures in mTBI patients, such as initial encoding and attention, are susceptible to stereotype threat. Kit et al. (2014) induced this result by having participants read material that emphasized the stereotype that mTBI leads to cognitive difficulties. Stereotype threat is often called diagnosis threat when participants are made aware of their diagnosis and told that others with the same diagnosis typically perform poorly on such tests (Suhr \& Gunstad, 2002). Diagnosis threat has been shown in some studies to negatively impact mTBI patients' attention and working memory (Suhr \& Gunstad, 2005).

The nocebo effect is the experience of symptoms due to beliefs and expectations of negative consequences from a benign stimulus (Vanderploeg, Belanger, \& Kaufmann, 2014). Iverson, Brooks, Ashton and Lange (2010) found that mTBI patients reported significantly more quantity and severity of PCS symptoms when given a checklist of possible symptoms compared to being assessed with open-ended questions. One hypothesis they used to explain this finding is the nocebo effect. Participants given the 
checklist may be led to believe that mTBI typically causes these symptoms, thus causing them to actually feel the symptomology themselves (Iverson et al., 2010).

It is unclear if the causal mechanisms for the psychosocial phenomena of stereotype threat, diagnosis threat, and the nocebo effect are different, similar, or the same. Stereotype threat is currently thought to be multiply mediated by extra pressure to succeed, threats to self-integrity, and priming (Spencer, Logel, \& Davies, 2016). A number of factors hypothesized to mediate underperformance seen in diagnosis threat including effort, anxiety, depression (Suhr \& Gunstad, 2005), pre-existing injury beliefs, and suggestibility (Carter-Allison, Potter, \& Rimes, 2016) have not yet been well supported. The mechanism for nocebo effect has been thought to include factors such as participant expectations (Barsky, Saintfort, Rogers, \& Borus, 2002; Nestoriuc, Orav, Liang, Horne, \& Barsky, 2010), depression, and anxiety (Barsky et al., 2002), but is not yet well understood (Ruan \& Kaye, 2015).

It is important to understand how psychosocial phenomena such as stereotype threat, diagnosis threat, and the nocebo effect may be induced to negatively affect the cognitive functioning and subjective symptomology of mTBI patients. Doing so can give us insight into how PCS is manifested and maintained as well as how PCS may be prevented or lessened for the mTBI patient.

The manifestation of PCS is one of several topics related to mTBI that have not been fully clarified. Yet many myths about mTBI have been developed without reliable empirical findings. These have formed and are perpetuated for a number of reasons, including poorly designed studies, conclusions being made based on insufficient 
evidence, complexity of the subject, and the hunger of the general public to understand questions that science cannot yet answer.

Some common myths include the ideas that mTBI patients always fully heal or never fully heal (Young, 2008). As discussed above, some patients do recover fully and others have long-term symptoms. There is heterogeneity amongst the reasons that are given for someone not recovering fully. However, one person's outcome can lead to premature assumptions about the course of mTBI in a seemingly similar patient.

Another myth is that multiple mTBIs inevitably lead to chronic traumatic encephalopathy (CTE), a neurodegenerative disorder with progressive tauopathy that presents with changes in memory, personality, behavior, and eventual motor difficulties. However, there have only been a total of 262 confirmed cases of CTE (Maroon et al., 2015). Baugh, Robbins, Stern, \& McKee (2014) explain that the field does not currently have a good understanding about which factors beyond the history of repetitive head injury, such as personality and lifestyle, lead to CTE and which factors may be protective. They note how outlets, such as the media, overgeneralize the research done on professional athletes to the general population who likely differ in several significant ways. A review of CTE in retired athletes indicated that there is not enough evidence to conclude that repeated mTBI alone leads to any specific neurodegenerative disease (Karantzoulis \& Randolph, 2013).

The general public's interest in mTBI is increasing while scientific knowledge about mTBI is advancing, yet still requires a great deal of clarity. This increase in attention may have been catalyzed by factors such as ongoing military operations and the pathological diagnosis of CTE in former professional football players. Either 
independently or in response to the public's interest, many media programs have sensationalized unsubstantiated dangers of mTBI (DiFazio et al., 2015). Indeed, the media has been accused in the past of purposely instilling undue fear in the public to raise the amount of media attention (Klemm, Das, \& Hartmann, 2014).

Framing theory is the idea that information is both conveyed and understood through frames or schemas (Goffman, 1974, p. 21-26; Scheufele, 1999). Journalists and reporters use framing to deliver complex information in a limited amount of space or time (Cissel, 2012). Varying how an issue is framed in the media can influence how the audience thinks about the topic (Cissel, 2012; Scheufele \& Tewksbury, 2007), including health topics (Coleman, Thorson \& Wilkens, 2011). Which frame a person has been exposed to most recently has more of an effect on a person's opinion than the additive effect of repeated exposure (De Vreese \& Lecheler 2012). Few studies have been done about how the media uses framing on the topic of mTBIs (Bell \& Sanderson, 2016; Cassilo \& Sanderson, 2016; Kennard, McLellan, \& McKinlay, 2017). However, we do know that televised media programs are discussing, with certainty, aspects of the effects of mTBI that have little or no scientific support (Block, West, \& Goldin, 2016).

Americans have been shown to spend the largest percentage of their leisure time watching television (Bureau of Labor Statistics, 2014). Often, adults rely on news media for health information (Van Slooten, Friedman, \& Tanner, 2013). The Institute of Medicine (2004) defines health literacy in part as "the degree to which individuals can obtain, process, and understand the basic health information and services they need to make appropriate health decisions." Older adults have been shown to have lower levels of health literacy than younger adults (Kutner, Greenberg, Jin, \& Paulsen, 2006). 
Additionally, adults over the age of 55 spend more of their time watching television than does any other age group (Bureau of Labor Statistics, 2014).

One study showed that over half of the participants indicated getting the majority of their medical information from television shows such as Dr. Oz or House (Block et al., 2014). Block et al. (2016) noted that such shows provide information that is dramatized and inaccurate, leading to negative impacts such as stigma and self-doubt in those recovering from $\mathrm{mTBI}$.

Witthoff and Rubin (2013) demonstrated how televised information can affect participants' symptomology and attribution beliefs. Their results showed that participants who watched televised reports about the adverse effects of WiFi showed more symptomology, attributed these symptoms to $\mathrm{WiFi}$, and had more worry about the effects of WiFi on their health than did a control group after both groups received a sham exposure to a WiFi signal.

Studies have revealed that a patient's negative beliefs about the timeline, consequences, and personal control after an mTBI can significantly affect the patient's prospect of achieving a full recovery (Hou et al., 2012; Roth \& Spencer, 2013). It has also been shown that negative or positive beliefs about recovery from mTBI can be rooted in the patient through mediums such as informational literature in a waiting room (Waldron-Perrine, Tree, Spencer, Suhr, \& Bieliauskas, 2015).

In summary, patients' mTBI-related beliefs are predictive of how well they will recover. These beliefs may be affected by phenomena such as stereotype threat, diagnosis threat, and the nocebo effect, which may be induced by how media is framed when reporting about mTBI. As a result, mTBI patients may experience perceptions of 
impaired cognitive functioning and subjective symptomology of their injury beyond the effects expected from the neurometabolic injury. Those with lower functional health literacy may be more susceptible to this effect than those with higher levels of functional health literacy.

The present study was designed to ascertain if, as hypothesized, viewing a sensationalized media clip about mTBI can lower participants' cognitive performance (working memory and attention) and induce more severe PCS reporting when compared to a non-sensationalized media clip. This study had the potential to add to the body of knowledge that will eventually lead to prevention measures, such as increasing health literacy, that can in-turn reduce the emotional and financial costs that mTBI has on individuals and society.

\section{Present Study}

\section{Primary Hypotheses}

The present study aimed to discover if exposure to sensationalized media about mTBI can negatively affect participants with a history of mTBI. It was hypothesized that:

1. Participants exposed to the sensationalized clip would perform worse on a measure of working memory and attention than participants exposed to the non-sensationalized clip. The Paced Auditory Serial Addition Test (PASAT) was used for this measurement. It has been shown to be one of the more sensitive tools to detect the cognitive deficiencies such as those found in working memory and attention impairments with mTBI patients (Cicerone \& Azulay, 2002). 
2. Participants exposed to the sensationalized clip as opposed to the nonsensationalized clip would indicate more severe PCS symptoms as indicated by having higher scores on the Rivermead Post Concussion Symptoms Questionnaire (RPQ). The RPQ is one of the most commonly used measurements for subjective PCS severity (Laborey et al., 2014).

\section{Secondary Hypotheses}

Secondary hypotheses were made with the idea that this study could produce both exploratory and pilot data for future studies. It was hypothesized that:

1. Consistent with the literature (Kutner et al., 2006), we hypothesized that older adults in our study would perform lower on a test of functional health literacy than younger adults.

2. We hypothesized that functional health literacy would be shown to impact the influence of video type on the two dependent variables. 


\section{CHAPTER II}

\section{METHOD}

\section{Participants}

The University of Colorado Colorado Springs (UCCS) Institutional Review Board (IRB) approved all recruitment and investigational procedures for this study (Appendix A). Participants were then recruited between August 2016 to May 2017. Several recruitment resources were utilized, including the UCCS research registry and psychology study recruitment website, Sona. Recruitment flyers were also posted in the community. The study was described as an exploration of how the participants' ability to remember and rate information from a video clip may be affected by participating in cognitive assessments and psychological questionnaires. UCCS students were offered extra credit for their participation. Participants who were not UCCS students were offered $\$ 10$ for their participation. The study took an average total time of 45 to 60 minutes for each of the participants to complete.

To be eligible for the study, individuals had to be between the ages of 18 and 32 (younger adults) or 55 and older (older adults). Having these separate age groups allowed for the collection of pilot and exploratory data about potential effect size differences seen between age groups. Individuals remained eligible if they self-reported a history of sustaining at least one mTBI that did not occur within the last three months, to avoid including participants who may be actively recovering from an mTBI. Individuals were excluded if they self-reported ever having received a diagnosis of schizophrenia, bipolar 
disorder, autism, or moderate to severe TBI. Individuals were also excluded if they reported taking any medications that may have altered their thinking ability. Lastly, individuals from the research registry and community members who replied to the recruitment flyers were screened by phone. This screening was done with the Memory Impairment Screen by Telephone (MIS-T; measurement is described below). They were excluded if they scored five points or lower.

\section{Materials}

Memory Impairment Screen by Telephone (MIS-T). Lipton et al., (2003)

developed the MIS-T from the Memory Impairment Screen (MIS; Buschke et al., 1999). The MIS-T has four items and an interruption task that tests individuals' delayed free and cued recall. This telephone screening takes approximately four minutes. Individuals can score between 0 and 8 points. Higher scores on the MIS-T suggest higher cognitive functioning. Individuals had to score a minimum of 6 points to remain eligible. This cutoff was chosen because it offered a high balance of sensitivity (93\%) and specificity (72\%) ensuring that almost everyone who passed the screening was cognitively intact (Lipton et al., 2003).

Paced Auditory Serial Addition Test (PASAT). The PASAT was used to measure working memory and attention (Gronwall, 1977; Rao, Leo, Bernardin, \& Unverzagt, 1991; Rao, Leo, Haughton, St. Aubin-Faubert, \& Bernardin, 1989). As described by Strauss, Sherman, and Spreen (2006), this is an auditory test in which participants are instructed to consecutively add pairs of numbers that are presented at a specific rate without interruption. For example, the second number is added to the first number and the third number is added to the second; these sums are spoken aloud by the 
examinee. In other words, if the stimulus begins with " 5 " and " 3 ," the first correct answer is " 8 ." If the next stimulus is "4", the next correct answer is " 7 ," and so forth, for 61 stimuli (60 possible responses). The interstimulus interval (ISI) was decreased from three seconds on the first trial to two seconds on the second trial, which adds greater challenge to the participants. Total test time was approximately 10 to 15 minutes in which a maximum of 120 responses dispersed equally across trials may be given. The total number of correct responses was used as the main outcome variable (Strauss et al., 2006). Higher scores on the PASAT indicate higher levels of cognitive functioning. Raw scores were converted to z-scores for analyses using the mean and standard deviation of the sample.

Rivermead Post-Concussion Symptom Questionnaire (RPQ). The RPQ was developed by King, Crawford, Wenden, Moss, and Wade (1995) to measure the progression of mTBI and PCS symptoms. This questionnaire was used to ask the participants to indicate how often they have suffered from a list of 16 symptoms in the past 24 hours compared to the symptoms they may have experienced before the mTBI. Symptoms included items such as headache, restlessness, and fatigue. There was also space for participants to list two additional symptoms. Each symptom was rated on a five-point scale from $0=$ "Not experienced at all" to $4=$ "A severe problem." The symptom severity ratings were summed and used as the main outcome variable with a higher score indicative of more suffering. Raw scores were converted to z-scores for analyses using the mean and standard deviation of the sample.

\section{Patient Reported Outcomes Measurement Information System (PROMIS).}

The PROMIS computerized adaptive test (CAT) version 1.0 was used to assess the 
participants' symptoms of depression. This version was programmed to provide specific items based on the participants' responses to previous questions (Assessment Center, 2015). The participants were asked between four to 12 items. For example, participants may have been asked how often he or she has had trouble feeling close to people in the past seven days. The participants chose from the responses "never," "rarely," "sometimes," "often," or "always." Item response theory (IRT) is the metric used to score the assessment and scores across participants are comparable. The program calculates a raw score and a $t$-score for individual participants. Higher scores indicate more severe depressive symptoms. The $t$-scores were converted to z-scores using the sample mean and standard deviation.

Posttraumatic Stress Disorder Checklist for DSM-5 (PCL-5). The PCL-5 was created by Weathers, Litz, Keane, Palmieri, and Marx (2013). It measures posttraumatic stress disorder (PTSD) symptomology. This was a 20-item self-assessment instrument. Participants were asked to rate each item on a scale of 0 to 4 . Higher summed scores indicate greater PTSD symptom severity. Raw scores were converted to z-scores for analyses using the mean and standard deviation of the sample.

Shortened Test of Functional Health Literacy in Adults (S-TOFHLA). The STOFHLA was developed by Baker, Williams, Parker, Gazmararian, \& Nurss (1999) to measure health literacy. This instrument contains two reading comprehension passages. One passage was written at a fourth grade reading level while the other passage was written at a tenth grade reading level. Both passages have a number of missing words. Participants were asked to select the correct word from four options and were given 1 point for each correct response for a maximum of 36 points. Higher summed scores 
indicate higher levels of health literacy. For ease of use, outcome data is generally divided into three categories of functional literacy: inadequate (0-16), adequate (17-22) and functional (23-36). For this study, raw scores were converted to z-scores for analyses using the sample mean and standard deviation.

Final Questionnaire - Rating. This five-item questionnaire was designed by the researchers to capture the participants' opinions about various aspects of the video, such as production quality and minimum education needed to understand the video.

Participants were asked to rate each item on a five-level Likert scale. Responses included "strongly agree," "somewhat agree," "neither agree nor disagree," "somewhat disagree," "strongly disagree." Point values from 1 to 5 were assigned to each response with 1 indicating "strongly disagree" and 5 indicating "strongly agree." The opinion we were most interested in for the purposes of this study was indicated by the responses to the statement "I believed what was said in the video." Raw scores were converted to z-scores for analysis using the sample mean and standard deviation.

\section{Procedures}

After consenting, qualified participants were randomly assigned to one of the two levels of the main independent variable, the Sensationalized or Non-Sensationalized Media group. The only difference between these two groups was the video clip that the participants watched. Each video clip was about 2 minutes long.

The principal investigator (PI) performed a comprehensive search for videos pertaining to mTBI that were available through YouTube.com and various news websites. These videos were rated by the PI and an independent rater on a number of variables, including production quality, believability, attire of presenters, lighting, 
primary topic, and the rater's opinion of the minimum education level needed to understand the message. The 10 video clips that were most closely matched on these variables were then rated by the PI and two colleagues with a slightly modified version of a questionnaire developed by Hoffman \& Justicz (2016; Appendix B). This questionnaire allows for a sensationalism rating and a scientific rating to be calculated for each clip. The video clip with the largest difference between the sensationalism and scientific ratings that had the highest sensationalism rating was selected for the Sensationalized Media group. The video clip with the largest difference between the scientific and sensationalism ratings that had the highest scientific rating was selected for the Nonsensationalized Media group.

Participants from both groups filled out their demographic information and then were given the S-TOFHLA. Participants were then reminded that they would watch a video clip and then participate in a cognitive assessment and several psychological questionnaires, which would be followed by a factual multiple-choice questionnaire and a rating questionnaire about the video.

The participants were then shown either the sensationalized or nonsensationalized media clip. After viewing the video clip, the participants were administered the PASAT, RPQ, PROMIS, and PCL-5. Following the questionnaires, participants were given the video rating sheet as well as a factual multiple-choice questionnaire about the video to correspond with the instructions they had been given at the start of their participation in the study. Participants were then debriefed and compensated. 


\section{CHAPTER III}

\section{DATA ANALYSIS}

\section{Power Analysis}

A power analysis was performed in $\mathrm{R}$ version 3.2.3 ( $\mathrm{R}$ Core Team, 2015) to determine the required number of participants. Power $(1-\beta)$ was set at .8 and alpha $(\alpha)$ was set at .0375 using the False Discovery Rate correction method (Benjamini \& Hochberg, 1995). A negative medium effect size of sensationalized media on PASAT performance was predicted (Cohen's $d=-0.5$ ) based on stereotype threat, diagnosis threat, and nocebo effect literature (Kit, et al., 2014; Lamont, Swift, \& Abrams, 2015; Nevelsteen, Legros, \& Crasson, 2007).

Kit, et al. (2014) found that their TBI-heightened threat condition had an effect size of Cohen's $d=-0.8$ on participants' attention. Lamont et al. (2015) conducted a meta-analysis of age based stereotype threat (ABST). They found that ABST had an effect size of Cohen's $d=-0.21$ for memory and Cohen's $d=-.68$ for cognitive performance. Nevelsteen et al. (2007) found the effect size of their nocebo condition to be Cohen's $d=-0.4$ on working memory. These effect sizes were averaged to predict the effect size of the current study to be Cohen's $d=-0.5$.

A total of 128 participants, 64 for each level of the primary independent variable, were suggested to achieve the desired statistical power when $\alpha=.05$. A total of 140 participants, 70 for each level of the primary independent variable, were needed to 
achieve the desired statistical power when $\alpha=.0375$. Therefore, a minimum goal of 128 participants, 64 for each level of the primary independent variable was set, but a total of 140 participants was desired. A total of 102 participants, 51 for each level of the independent variable, were ultimately recruited.

\section{Traditional Analysis}

All traditional and Bayesian analyses were done using $\mathrm{R}$ version 3.4.0 (R Core Team, 2017) and all analyses used z-scores for PASAT, RPQ, S-TOFHLA, PROMIS, PCL-5, and believability ratings. The traditional analyses consisted of two independent samples Welch's $t$-tests, one for each of the dependent variables. Both $t$-tests used the same independent variable, video type. One $t$-test used totaled PASAT scores as the dependent variable while the other $t$-test used totaled RPQ scores as the dependent variable. Analyses were run to examine differences between the Sensationalized Media group and Non-sensationalized Media group on the dependent variables. We used the statistical package 'effsize' (version 0.7.1; Torchiano, 2017) to determine Cohen's $d$.

Two multiple linear regression analyses were conducted to control for any potential confounds caused by group discrepancies in age, health literacy, or symptoms of depression or PTSD. Both analyses used the S-TOFHLA, PROMIS, PCL-5, and interactions with video type as predictor variables. One regression used PASAT score as the dependent variable while the other regression used RPQ score as the dependent variable. We used the statistical package 'gvlma' (version 1.0.0.2; Pena \& Slate, 2014) to determine whether our data met the assumptions for these linear regression models. 


\section{Bayesian Analysis}

Bayesian analysis was used so that we could incorporate our previous knowledge about the effect of sensationalized media on cognitive functioning (Kit, et al., 2014; Lamont et al., 2015; Nevelsteen et al., 2007) into the results. The priors used in these analyses are listed in Table 1. We conducted two Bayesian $t$-tests to examine the effect of video type on PASAT and RPQ scores. Additionally, we conducted two Bayesian linear regression analyses. The same predictor and outcome variables used in the traditional regression analyses were used in the Bayesian analyses. Finally, we conducted one secondary Bayesian $t$-test and two secondary Bayesian regression analyses to identify avenues for further exploration in future studies. The secondary $t$-test was carried out to see if there was a difference between younger and older adults on S-TOFHLA scores. The secondary regression analyses incorporated the three-way interaction effect between Age, S-TOFHLA, and video type as well as the interaction between believability and video type. The same priors used for the primary Bayesian regression analyses were used for the secondary regression analyses. The dependent variables were again PASAT scores and RPQ scores.

We used the statistical software packages 'brms' (version 1.7.0; Bürkner, 2017) and 'rstan' (version 2.15.1; Stan Development Team, 2017) for these analyses. The Bayesian analyses utilized four chains, each with 2000 iterations (1000 warmup samples were discarded from each chain), for a total of 4000 post-warmup samples. Successful convergence was met when $\hat{R}$ (potential scale reduction factor) values were close to 1 ( \pm $.01)$. Posterior predictive checks were run to visually examine whether the posterior 
distribution provided a reasonable match to the actual data, therefore confirming that an acceptable model was used.

Table 1

Prior Distributions for Predictors used in Bayesian Analyses

\begin{tabular}{lcc}
\hline Variable & PASAT & RPQ \\
\hline Video type & $\mathrm{N}(-0.5,0.5)$ & $\mathrm{N}(0.5,0.5)$ \\
Age & $\mathrm{N}(-0.4,0.5)$ & $\mathrm{N}(0.01,1)$ \\
S-TOFHLA & $\mathrm{N}(0.02,1)$ & $\mathrm{N}(-0.01,1)$ \\
PROMIS & $\mathrm{N}(-0.2,1)$ & $\mathrm{N}(-0.2,1)$ \\
PCL-5 & $\mathrm{N}(-0.2,1)$ & $\mathrm{N}(0.1,1)$ \\
\hline
\end{tabular}

Note: All priors are weakly informative priors except for PASAT on video type, which is an informative prior based on the existing literature. 


\section{CHAPTER IV}

\section{RESULTS}

\section{Participants}

Participant demographics can be found in Table 2 and participant mTBI history can be found in Table 3 .

Table 2

Participant Demographics

\begin{tabular}{|c|c|}
\hline Variable & Value \\
\hline Age; $M(S D)$ & $37.15(22.61)$ \\
\hline Sex; (Female) $n(\%)$ & $66(66.30)$ \\
\hline Handedness ${ }^{\mathrm{a}}$; (Right) $n(\%)$ & $52(86.67)$ \\
\hline Years of education ${ }^{\mathrm{a}} ; M(S D)$ & $15.57(2.13)$ \\
\hline \multicolumn{2}{|l|}{ Ethnicity ${ }^{\mathrm{a}}$} \\
\hline Not Hispanic / Latino; $n(\%)$ & $52(86.67)$ \\
\hline Hispanic / Latino; $n(\%)$ & $4(6.67)$ \\
\hline Unknown; $n(\%)$ & $4(6.67)$ \\
\hline \multicolumn{2}{|l|}{ Race $^{\mathrm{a}}$} \\
\hline Caucasian; $n(\%)$ & $50(83.30)$ \\
\hline Black / African-American; $n(\%)$ & $4(6.67)$ \\
\hline Asian; $n(\%)$ & $3(5.00)$ \\
\hline Native American; $n(\%)$ & $2(3.33)$ \\
\hline Other; $n(\%)$ & $1(1.67)$ \\
\hline
\end{tabular}

A traditional Welch's $t$-test revealed no significant differences between the Sensationalized $(M=82.02, S D=20.6)$ and Non-sensationalized $(M=79.76, S D=$ 19.13) Media groups in relation to PASAT scores, $t(99.46)=0.57, p=.57$. The standardized mean difference between groups was very small (Cohen's $d=0.11,95 \% \mathrm{CI}$ 
Table 3

Participant Concussion History

\begin{tabular}{lc}
\hline Variable & Value \\
\hline Months from last mTBI ${ }^{\mathrm{a}}$; M (SD) & $55.91(52.62)$ \\
Lifetime mTBIs & \\
One; n (\%) & $57(55.88)$ \\
Two; n (\%) & $27(26.47)$ \\
Three; n (\%) & $12(12.00)$ \\
Four or more; n (\%) & $6(5.88)$ \\
\hline
\end{tabular}

${ }^{\mathrm{a}}$ Values account for $60(58.2 \%)$ of the 102 participants.

$[-0.28,0.51])$. Similarly, a traditional Welch's $t$-test revealed no significant differences between the Sensationalized $(M=15.88, S D=11.8)$ and Non-sensationalized $(M=$ $17.69, S D=11.77)$ Media groups in relation to RPQ scores $t(100)=-0.77, p=.44$. The standardized mean difference between groups was also very small (Cohen's $d=-0.15$, $95 \%$ CI $[-0.55,0.24])$.

Examination of results of the traditional analysis regressing PASAT scores onto S-TOFHLA, PROMIS, PCL-5, age category and the interactions between these variables and video type indicated that age category $(\beta=-0.74, S E=0.28, p<.05)$ was a significant predictor of PASAT performance. S-TOFHLA performance $(\beta=0.21, S E=$ $0.13, p=.11)$ showed a small effect on PASAT scores, but was not statistically significant. PROMIS scores $(\beta=0.01, S E=0.18, p=.96)$, PCL-5 $\operatorname{scores}(\beta=-0.02, S E=$ $0.15, p=.89)$, and video type $(\beta=0.02, S E=0.22, p=.92)$ were found to be poor predictors of PASAT performance. Results for the interactions between video type and the other independent variables did not indicate any reliable predictors of PASAT score (see Table 4). 
Table 4

Interaction Effects for PASAT Traditional Regression

\begin{tabular}{lccc}
\hline Interaction & $\beta$ & $S E$ & $p$-value \\
\hline Video type $^{\mathrm{a}}$ x S-TOFHLA & 0.20 & 0.19 & .30 \\
Video type $^{\mathrm{a}}$ x PROMIS & -0.25 & 0.23 & .27 \\
Video type $^{\mathrm{a}}$ x PCL-5 & 0.35 & 0.23 & .13 \\
Video type $^{\mathrm{a}} \times$ Age & 0.35 & 0.42 & .41 \\
\hline
\end{tabular}

Note: $\beta=$ standardized regression coefficient; $S E=$ standard error. ${ }^{\text {a }}$ Reference group is non-sensationalized media group. ${ }^{b}$ Reference group is younger age group.

Inspection of the results of a traditional analysis regressing RPQ scores onto STOFHLA, PROMIS, PCL-5, age category, and the interactions between these variables and video type revealed that PCL-5 $(\beta=0.54, S E=0.13, p<.05)$ was a strong predictor of RPQ scores. PROMIS $(\beta=0.16, S E=0.15, p=.30)$, age category $(\beta=-0.11, S E=$ $0.24, p=.70)$, and video type $(\beta=-0.17, S E=0.19, p=.37)$ were shown to have a very small effect on RPQ scores that was not reliably above 0 . S-TOFHLA $(\beta=-0.01, S E=$ $0.11, p=.93)$, was not found to be a strong predictor of PRQ performance. Results for the interactions between video type and the other independent variables did not indicate any reliable predictors of RPQ score (see Table 5).

Table 5

Interaction Effects for RPQ Traditional Regression

\begin{tabular}{lccc}
\hline Interaction & $\beta$ & $S E$ & $p$-value \\
\hline Video type $^{\mathrm{a}} \times$ S-TOFHLA & 0.10 & 0.16 & .54 \\
Video type ${ }^{\mathrm{a}} \times$ PROMIS & 0.22 & 0.19 & .23 \\
Video type $^{\mathrm{a}} \times$ PCL-5 & -0.11 & 0.19 & .57 \\
Video type $^{\mathrm{a}} \times$ Age & 0.05 & 0.36 & .89 \\
\hline
\end{tabular}

Note: $\beta=$ standardized regression coefficient; $S E=$ standard error. ${ }^{\text {a Reference group is }}$ non-sensationalized media group. ${ }^{\mathrm{b}}$ Reference group is younger age group. 


\section{Bayesian Analyses}

With Bayesian analyses, we were able to combine our prior knowledge from the research literature with our actual data collected during this study to obtain an updated estimate of the distribution of the effects called a posterior distribution. Our informative prior estimate of the effect of sensationalized media on PASAT scores was a normal distribution with $M=-0.5$ and $S D=0.5$. In other words, we used the existing literature to estimate that the most likely effect of sensationalized media was to decrease PASAT scores by half a standard deviation, but that effect sizes roughly between -2 and +1 SDs were also plausible (see Figure 1). The posterior distribution arising from this analysis had a mean value $(\beta)$ of $0.04(S E=0.19,95 \%$ Credible Interval $(\mathrm{CI})[-0.33,0.40]$; see Figure 1), suggesting a very small effect of video type on PASAT scores that was not reliably different from 0 . The results from this Bayesian $t$-test were consistent with and more precise than the findings of the traditional $t$-test discussed above. Lastly, examination of this analysis found it approximately four times more likely (Evidence ratio $=4.02$ ) that the null hypothesis is true (the effect of being exposed to sensationalized media is 0 ) compared to the alternative hypothesis (the effect of being exposed to sensationalized media is not 0 ).

Likewise, our weakly informative prior estimate of the effect of sensationalized media on RPQ scores was a normal distribution with $M=0.5$ and $S D=0.5$. The posterior distribution arising from this analysis had a mean $(\beta)$ of $-0.03(S E=0.19,95 \%$ CI [-0.40, 0.33]; see Figure 2) suggesting a very small effect of video type on RPQ scores. The results from this Bayesian $t$-test were consistent with the findings from the traditional $t$ test discussed above. Again, examination of this analysis found it over four times more 


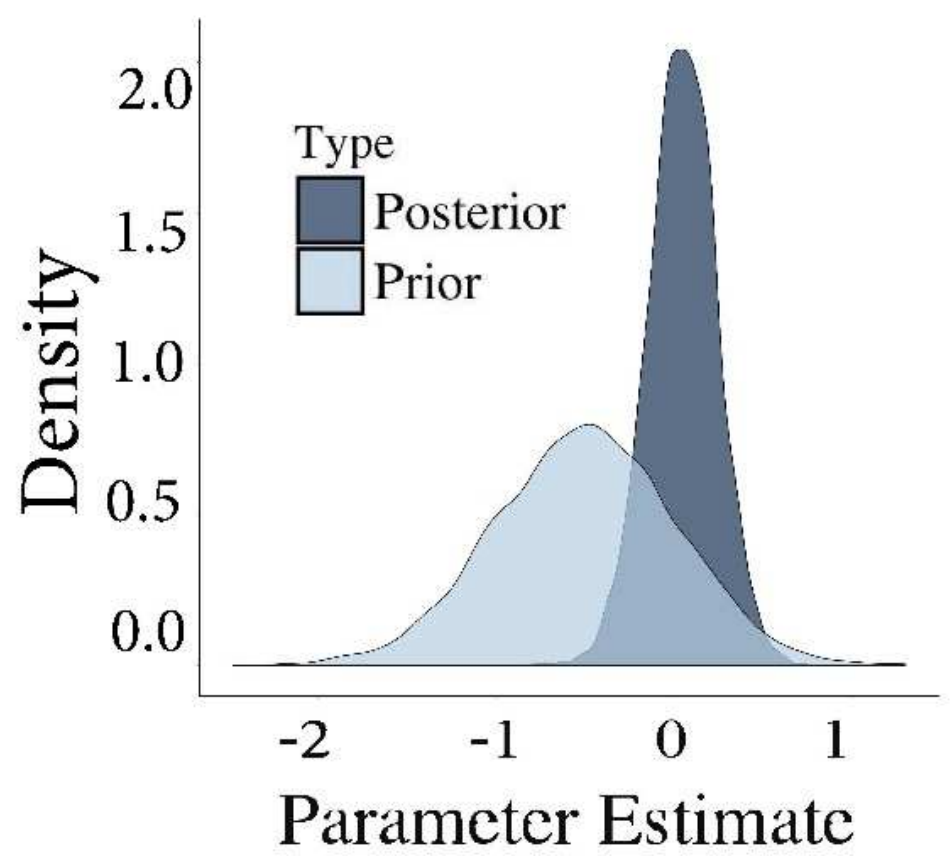

Figure 1. Plot of prior and posterior distributions of parameter estimates for the effect of sensationalized media on PASAT performance.

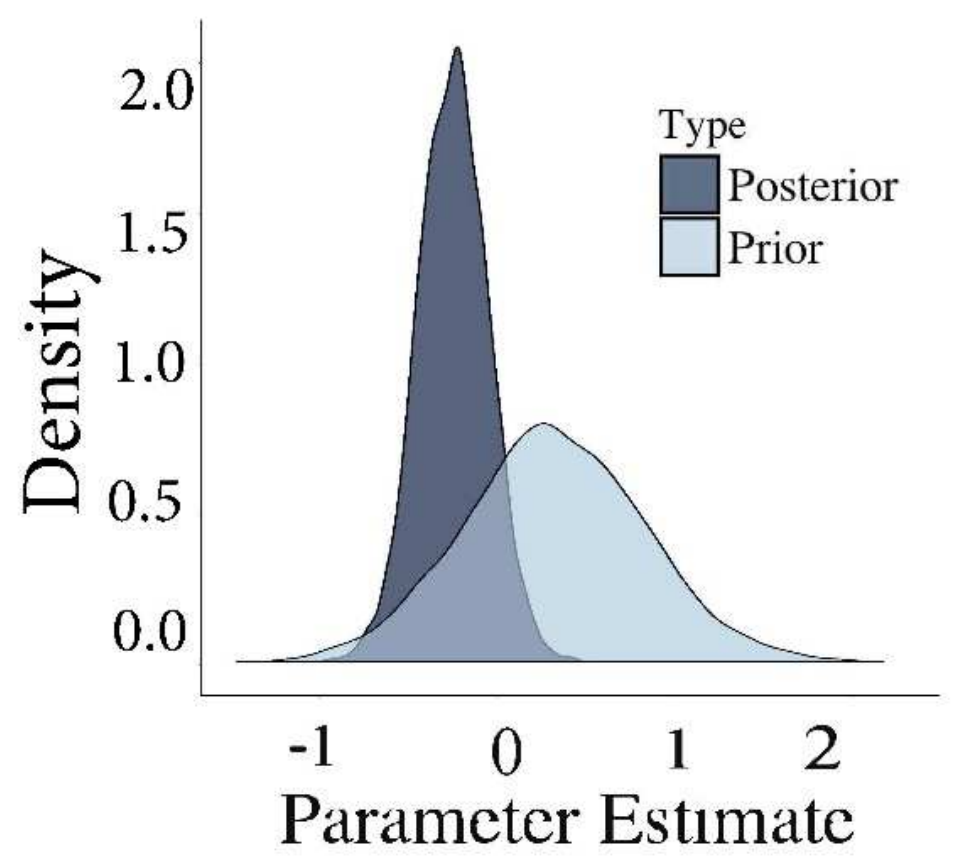

Figure 2. Plot of prior and posterior distributions of parameter estimates for the effect of sensationalized media on RPQ score. 
likely (Evidence ratio $=4.69)$ that the null hypothesis is true (the effect of being exposed to sensationalized media is 0 ) compared to the alternative hypothesis (the effect of being exposed to sensationalized media is not 0 ).

The results of a Bayesian regression, which regressed PASAT onto S-TOFHLA, PROMIS, PCL-5, age category, and the interactions between these variables and video type, were also consistent with the results of the traditional regression analysis in that the Bayesian regression analysis indicated that age category $(\beta=-0.63, S E=0.25,95 \%$ CI [$1.10,-0.12])$ was a moderate to strong predictor of PASAT performance. Consistent with the traditional regression, S-TOFHLA $(\beta=0.22, S E=0.13,95 \%$ CI $[-0.02,0.47])$ was found to have a small effect on the PASAT that was $96 \%$ likely to be greater than 0 . PROMIS $(\beta=0.02, S E=0.18,95 \%$ CI $[-0.34,0.37])$, PCL-5 $(\beta=-0.03, S E=0.15,95 \%$ CI $[-0.33,0.27])$, and video type $(\beta=0.12, S E=0.21,95 \%$ CI $[-0.29,0.54])$ were again shown to be weak predictors of PASAT performance. Results for the interactions between video type and the other predictor factors did not indicate any strong predictors of PASAT score (see Table 6).

Table 6

Interaction Effects for PASAT Bayesian Regression

\begin{tabular}{lccc}
\hline Interaction & $\beta$ & $S E$ & $95 \%$ CI \\
\hline Video type $^{\mathrm{a}} \times$ S-TOFHLA & 0.19 & 0.20 & {$[-0.21,0.57]$} \\
Video type $^{\mathrm{a}}$ x PROMIS & -0.27 & 0.23 & {$[-0.73,0.18]$} \\
Video type $^{\mathrm{a}}$ x PCL-5 & 0.35 & 0.23 & {$[-0.09,0.81]$} \\
Video type $^{\mathrm{a}} \times$ Age & 0.20 & 0.41 & {$[-0.62,0.99]$} \\
\hline
\end{tabular}

Note: $\beta=$ standardized regression coefficient; $S E=$ standard error; $\mathrm{CI}=$ credible interval. ${ }^{a}$ Reference group is non-sensationalized media group. ${ }^{b}$ Reference group is younger age group. 
Examination of the results of a similar Bayesian regression, which regressed RPQ onto S-TOFHLA, PROMIS, PCL-5, age category, and the interactions between these variables and video type were also consistent with the results found in the traditional regression analysis. Inspection of the results indicated that PCL-5 ( $\beta=0.54, S E=0.13$, $95 \%$ CI $[0.29,0.79])$ was a strong predictor of RPQ scores. Again, consistent with the results of the traditional regression analysis, PROMIS $(\beta=0.16, S E=0.15,95 \%$ CI [$0.41,0.44])$ had a very small effect on RPQ that was not reliably different from 0 . Age category $(\beta=-0.06, S E=0.24,95 \%$ CI $[-0.52,0.41])$, video type $(\beta=-0.08, S E=0.17$, 95\% CI $[-0.43,0.26])$, and S-TOFHLA $(\beta=0.00, S E=0.11,95 \% \mathrm{CI}[-0.21,0.26])$ were given point estimates of effect size even smaller than the traditional analyses and were not found to be strong predictors of RPQ scores. Results for the interactions between video type and the other predictor factors did not indicate any strong predictors of RPQ score (see Table 7).

Table 7

Interaction Effects for RPQ Bayesian Regression

\begin{tabular}{lccc}
\hline Interaction & $\beta$ & $S E$ & $95 \%$ CI \\
\hline Video type $^{\mathrm{a}} \times$ S-TOFHLA & 0.09 & 0.16 & {$[-0.22,0.41]$} \\
Video type $^{\mathrm{a}} \times$ PROMIS & 0.22 & 0.19 & {$[-0.15,0.59]$} \\
Video type $^{\mathrm{a}} \times$ PCL-5 & -0.11 & 0.20 & {$[-0.49,0.26]$} \\
Video type $^{\mathrm{a}} \times$ Age & -0.06 & 0.35 & {$[-0.73,0.61]$} \\
\hline
\end{tabular}

Note: $\beta=$ standardized regression coefficient; $S E=$ standard error; $\mathrm{CI}=$ credible interval.

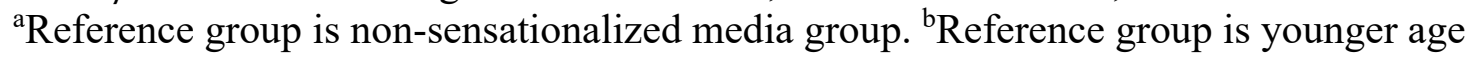
group.

\section{Secondary Analysis}

A Bayesian $t$-test was carried out to examine if older adults, when compared to younger adults, had lower scores on the S-TOFHLA. Results indicated that the older 
group $(M=35.10, S D=1.08)$ and younger group $(M=35.18, S D=0.91)$ were not reliably different from one another $(\beta=-0.04, S E=0.41,95 \% \mathrm{CI}[-0.62,0.99])$ on $\mathrm{S}$ TOFHLA scores.

Potential interaction effects of Age, S-TOFHLA, believability, and video type were examined with Bayesian regression analysis. Results indicated that these interactions were not reliable of PASAT performance (see Table 8) and RPQ scores (see Table 9).

Table 8

Secondary Interaction Effects for PASAT Bayesian Regression

\begin{tabular}{|c|c|c|c|}
\hline Variables & $\beta$ & $S E$ & $95 \% \mathrm{CI}$ \\
\hline $\mathrm{VT}^{\mathrm{a}}$ & 0.14 & 0.15 & {$[-0.25,0.54]$} \\
\hline $\mathrm{Age}^{\mathrm{b}}$ & -0.61 & 0.25 & {$[-1.1,-0.11]$} \\
\hline S-TOFHLA & 0.23 & 0.19 & {$[-0.13,0.61]$} \\
\hline Believability & -0.09 & 0.14 & {$[-0.69,0.73]$} \\
\hline $\mathrm{VT}^{\mathrm{a}} \times \mathrm{S}$ S-TOFHLA & -0.05 & 0.28 & {$[-0.61,0.49]$} \\
\hline $\mathrm{VT}^{\mathrm{a}} \times \mathrm{Age}^{\mathrm{b}}$ & 0.02 & 0.37 & {$[-0.69,0.73]$} \\
\hline $\mathrm{VT}^{\mathrm{a}} \mathrm{x}$ Believability & 0.22 & 0.19 & {$[-0.16,0.60]$} \\
\hline Age $^{b} x$ S-TOFHLA & -0.02 & 0.25 & {$[-0.52,0.47]$} \\
\hline VT $^{\mathrm{a}} \times \mathrm{Age}^{\mathrm{b}} \times$ S-TOFHLA & 0.50 & 0.39 & {$[-.26,1.26]$} \\
\hline
\end{tabular}

Note: $\beta=$ standardized regression coefficient; $S E=$ standard error; $\mathrm{CI}=$ credible interval. ${ }^{a}$ Reference group is non-sensationalized media group. ${ }^{b}$ Reference group is younger age group. 
Table 9

Secondary Interaction Effects for RPQ Bayesian Regression

\begin{tabular}{|c|c|c|c|}
\hline Variables & $\beta$ & $S E$ & $95 \% \mathrm{CI}$ \\
\hline $\mathrm{VT}^{\mathrm{a}}$ & 0.06 & 0.22 & {$[-0.35,0.50]$} \\
\hline $\mathrm{Age}^{\mathrm{b}}$ & -0.23 & 0.26 & {$[-0.76,0.30]$} \\
\hline S-TOFHLA & -0.17 & 0.21 & {$[-0.57,0.24]$} \\
\hline Believability & 0.04 & 0.15 & {$[-0.27,0.34]$} \\
\hline $\mathrm{VT}^{\mathrm{a}} \times \mathrm{S}$-TOFHLA & 0.32 & 0.31 & {$[-0.31,0.94]$} \\
\hline $\mathrm{VT}^{\mathrm{a}} \mathrm{x}$ Age $^{\mathrm{b}}$ & -0.46 & 0.39 & {$[-1.21,0.31]$} \\
\hline $\mathrm{VT}^{\mathrm{a}} \mathrm{x}$ Believability & -0.04 & 0.21 & {$[-0.45,0.37]$} \\
\hline $\operatorname{Age}^{b} \times$ S-TOFHLA & 0.24 & 0.27 & {$[-0.31,0.76]$} \\
\hline VT $^{\mathrm{a}} \times \mathrm{Age}^{\mathrm{b}} \times \mathrm{S}$-TOFHLA & -0.60 & 0.42 & {$[-1.44,0.23]$} \\
\hline
\end{tabular}

Note: $\beta=$ standardized regression coefficient; $S E=$ standard error; $\mathrm{CI}=$ credible interval; $\mathrm{VT}=$ video type. ${ }^{\mathrm{a}}$ Reference group is non-sensationalized media group. ${ }^{\mathrm{b}}$ Reference group is younger age group. 


\section{CHAPTER V}

\section{DISCUSSION}

The general public is currently interested in the topic of mTBI and the news media is one place where they turn for health information (Block et al., 2014). News media can be framed in ways to draw more viewers and may or may not be completely accurate or scientifically based (DiFazio et al., 2015). We do not know the effect that framing media about mTBI in this way has on individuals with a history of mTBI. We are concerned that sensationalized media may induce psychosocial phenomena such as stereotype threat, diagnosis threat, or the nocebo effect in people who have a history of mTBI, resulting in lowered cognitive functioning and higher PCS symptomology. The primary purpose of this study was to see if media framed in a sensationalized manner as opposed to a non-sensationalized manner could negatively affect individuals with a history of mTBI.

Based on literature about stereotype threat, diagnosis threat, and the nocebo effect, we hypothesized that those exposed to sensationalized media, as opposed to nonsensationalized media, would perform worse on a test of working memory and attention and report more severe PCS symptomatology. Our results from Bayesian regression analyses were consistent with results from our traditional regression analyses. Participants who scored in the average range for our sample on assessments of health literacy, depression, and PTSD symptoms - and who encountered the sensationalized 
media - were affected to a small extent both in terms of working memory and attention and reporting PCS symptoms.

Based on a number of previous psychology studies (Kit, et al., 2014; Lamont et al., 2015; Nevelsteen, et al., 2007), we estimated a prior effect size of sensationalized media to be Cohen's $d=-0.5, S D=0.5$ for cognitive functioning (memory and attention) and Cohen's $d=0.5, S D=0.5$ for reporting PCS symptoms. These are considered medium effect sizes with standard deviations large enough to allow effects in the opposite direction as hypothesized. The literature that was used to estimate our effect size all included studies looking at the effect of stereotype threat (Lamont et al., 2015), diagnosis threat (Kit, et al., 2014), or the nocebo effect (Nevelsteen, et al., 2007). The current study was not meant to replicate their findings as there are many differences between the current study and the cited studies. For example, Lamont et al. (2015) was looking at the effect of age based stereotype threat. Kit et al. (2014) included participants with moderate head injuries. The research by Nevelseen et al. (2007) focused on a sham exposure to a magnetic field. These studies were chosen to give us an estimate of the effect size we may expect to find in the current study.

We used Bayesian analysis to incorporate these estimates (our prior knowledge), into our calculations. The results of this Bayesian $t$-test analysis indicated that a more accurate estimate of the effect of sensationalized media is Cohen's $d=0.04, S D=0.19$, $[95 \% \mathrm{CI}=-0.33,0.40]$ for cognitive functioning and Cohen's $d=-0.03, S D=0.19[95 \%$ $\mathrm{CI}=-0.40,0.33]$ for PCS symptom severity. In other words, a credible estimate for the effect of sensationalized media on cognitive functioning could be anywhere between a negative third of a standard deviation to almost a half of a standard deviation increase in 
PASAT scores. A credible estimate for the effect of sensationalized media on reporting PCS symptoms could be anywhere between almost a negative half of a standard deviation to a positive third of a standard deviation. Therefore, the prior effect size estimates of $d=$ 0.50 - which were based on previous findings - are not considered credible estimates after incorporating the data collected in our study into the analyses.

One potential reason that the prior estimates were inaccurate, even though they were based on previous literature about stereotype threat, diagnosis threat, and the nocebo effect, may be due to the file drawer problem (Rosenthal, 1979). This concept is based on the fact that many journals only publish results that are found to be statistically significant based on an arbitrary cut-off of $p<.05$. Therefore, there may have been numerous previous studies showing small to negligible effects that were not included in our estimates because they had never been published. Another potential explanation is that that our media clip framed in a sensationalized manner did not induce stereotype threat, diagnosis threat, or the nocebo effect.

Even though sensationalized media was not found to have strong direct effects on PASAT and RPQ scores, two other variables did. Consistent with prior literature, (Crawford,1998; Vanotti, 2016) belonging to the older age group (55 years and older, as opposed to the younger (18 to 32 years old) age group, produced a medium negative effect on PASAT scores that was credibly lower than 0 . That is, those in the older age group generally performed a little over a half of a standard deviation below those in the younger age group. However, this was not the case when looking at the effect in the sensationalized media group, which was imprecisely estimated. Increased precision can 
be obtained with additional data and would likely show a similar effect of age on PASAT scores.

Also consistent with prior literature (Silverberg \& Iverson, 2011), PCL-5 scores were found to have a medium effect on reporting PCS symptoms that was credibly above 0. Those who reported more PTSD symptomology tended to report more PCS symptomology when watching the non-sensationalized video. The effect of PCL-5 scores on reporting PCS symptoms was not found to have the same magnitude or precision of effect on participants who watched the sensationalized video. In general, the link between PTSD reporting and PCS reporting may be due to the symptom overlap between the items on the RPQ and the PCL-5. It is also possible that participants misattributed symptoms, in this case of PTSD, to PCS (Silverberg and Iverson, 2011; Snell et al., 2015).

We came across some interesting effects while examining the interactions of our variables, using both Bayesian regression and traditional regression. As described above, there was a small and imprecisely estimated effect of sensationalized media alone or PCL-5 scores alone on PASAT scores. However, the interaction of sensationalized media and PCL-5 scores on PASAT scores created a small and imprecise effect size estimate. The effect of sensationalized media on PASAT scores increased by about a third of a standard deviation for every standard deviation change in PCL-5 score. So, the effect of the sensationalized video differed a moderate amount depending on the person's reported PTSD symptoms, with more PTSD symptoms related to a stronger effect of sensationalized media. We addressed the fact that this effect and the other interaction effects are not precise in the limitations and future directions section below. This result 
highlights the importance of considering how interactions may have affected the results of previous studies.

A similar interaction effect occurred with PROMIS scores, S-TOFHLA scores, and age category. When we look at the effect that sensationalized media has based on PROMIS scores, we see that for every standard deviation change in PROMIS scores, PASAT scores decreases by about a quarter of a standard deviation, which is considered a small and, in this case, imprecise effect. The effect of sensationalized media based on S-TOFHLA is to increase PASAT scores by almost a fifth of a standard deviation for every standard deviation change in S-TOFHLA scores. This is also a small and, in this case, imprecise effect. The effect of sensationalized media on older adults is to increase PASAT scores by 0.20 standard deviations over the younger adults, but this result is not credibly different from zero.

When we look at the reporting of PCS symptoms, we see that PROMIS scores have more of an effect on RPQ scores when the participants watched the sensationalized media clip compared to the non-sensationalized media clip. However, these results are again imprecise.

In our introduction, we proposed two hypotheses to be examined as secondary analyses. It is important to note that these hypotheses were meant to exploratory in nature.

Based on previous literature (Kutner et al., 2006) we had hypothesized that our older adult age group would perform lower on our assessment of health literacy than our younger age group. We performed a Bayesian $t$-test because we had far fewer older adults $(n=33)$ than younger adults $(n=69)$ and Bayesian analysis does not operate under 
the same assumptions as frequentist analyses. The older age group in our sample did not perform lower on the S-TOFHLA $(\beta=-.04, S E=0.25,95 \% \mathrm{CI}=[-0.55,0.44])$ compared to the younger age group in our sample. One of the reasons that this finding was not consistent with previous literature could have been that our older adult sample was highly educated $(M=15.57, S D=2.13)$, which may have affected their S-TOFHLA scores (Kutner et al., 2006). All of our participants had at least a high school degree, whereas $19 \%$ of participants in the study done by Kutner et al. (2006) had less than a high school degree or equivalent at the time of testing.

Another secondary hypothesis that we had proposed based on prior literature was that functional health literacy would be shown to impact the influence of video type on the two dependent variables. Our analyses with Bayesian regression showed that health literacy scores did not have a precise impact the effect the effect of sensationalized media on PASAT or RPQ scores. However, it did appear that in those who were exposed to sensationalized media, having a higher level of functional health literacy was more impactful on PASAT scores for older adults than younger adults. A similar pattern emerged with RPQ scores. Having a higher level of functional health literacy was more impactful for older than younger adults. Again, these results were imprecise.

A final exploratory analysis we conducted looked at whether the level to which participants believed what was said in the sensationalized video would moderate the effect of the video on the dependent variables. We found that the level of believability indicated by the participant was not a strong moderator of PASAT or RPQ scores.

Bayesian analysis is able to incorporate our prior knowledge about the effects of sensationalized media on cognitive functioning and PCS symptom severity with the data 
we collected from our participants for this study. Because we were able to use informative and weakly informative priors in our primary data analyses, we are able to obtain a more precise estimate of a posterior distribution than if we had used an uninformative prior (Matthews, Deary, Whiteman, \& Matthews, 2013). The posterior distribution represents how our prior knowledge has been updated by the data we collected for this study. The posterior distributions from this research project may be used as informative prior distributions for future studies looking at the effects of these variables.

\section{Limitations and Future Research}

This study had a number of limitations and areas for growth with future research. First, our sample size $(N=102)$ was small. Therefore, with more participants, some of our imprecise effects may be estimated more precisely and therefore hypotheses about why we found the results that we did could be made.

Second, our study did not differentiate between stereotype threat, diagnosis threat and nocebo effect. Future studies may give a closer look to the mechanisms underlying these phenomena to better understand their similarities and differences. Doing so will allow researchers to directly test which, if any, of these mechanisms may play a role in the development of PCS.

Third, our participants were restricted to a specific region and tended to be welleducated. Our results can be generalized to other populations with similar demographic characteristics. Caution should be used when generalizing our results to populations with demographic characteristics that are different from this study population. 


\section{Conclusion}

Despite concerns about possible iatrogenic effects of media reporting that sensationalizes the effects of concussion, the current results, when combined with prior information based on the nocebo effect and related literature, suggest that these effects on their own are negligible. With the information we have, it appears that S-TOFHLA scores and age were the best predictors of working memory and attention. Depression symptoms were the best predictor of PCS symptom reporting. Future studies should consider utilizing the posterior distributions found by this study when researching the effect of sensationalized media on cognitive performance and PCS symptom reporting. 


\section{REFERENCES}

American Psychiatric Association. (2013). Diagnostic and statistical manual of mental disorders (5th ed.). Washington, DC.

Assessment Center. (2015). PROMIS Depression Scoring Manual. Retrieved from https://www.assessmentcenter.net/documents/PROMIS Depression Scoring Manual.pdf

Baker, D. W., Williams, M. V, Parker, R. M., Gazmararian, J., \& Nurss, J. (1999). Development of a brief test to measure functional health literacy. Patient Education and Counseling, 38(1), 33-42. http://doi.org/10.1016/S07383991(98)00116-5

Barsky, A. J., Saintfort, R., Rogers, M. P., \& Borus, J. F. (2002). Nonspecific medication side effects and the nocebo phenomenon. Jama, 287(5), 622. https://doi.org/10.1001/jama.287.5.622

Baugh, C. M., Robbins, C. a., Stern, R. a., \& McKee, A. C. (2014). Current understanding of chronic traumatic encephalopathy. Current Treatment Options in Neurology, 16(9). https://doi.org/10.1007/s11940-014-0306-5

Belanger, H. G., Curtiss, G., Demery, J., Lebowitz, B. K., \& Vanderploeg, R. D. (2005). Factors moderating neuropsychological outcomes following mild traumatic brain injury: a meta-analysis. Journal of the International Neuropsychological Society: JINS, 11(3), 215-227. http://doi.org/10.1017/S1355617705050277

Bell, T. R., \& Sanderson, J. (2016). A Hit on American Football: A Case Study of Bottom-up Framing Through Op-Ed Readers' Comments. International Journal of Sport Communication, 9(4), 499-518.

Benjamini, Y. \& Hochberg, Y. (1995). Controlling the false discovery rate : a practical and powerful approach to multiple testing, Journal of the Royal Statistical Society, 57(1), 289-300.

Bigler, E. D. (2008). Neuropsychology and clinical neuroscience of persistent postconcussive syndrome. Journal of International Neuropsychological Society, 14(1), 1-22. http://doi.org/10.1017/s135561770808017x 
American Psychiatric Association. (2013). Diagnostic and statistical manual of mental disorders (5th ed.). Washington, DC.

Assessment Center. (2015). PROMIS Depression Scoring Manual. Retrieved from https://www.assessmentcenter.net/documents/PROMIS Depression Scoring Manual.pdf

Baker, D. W., Williams, M. V, Parker, R. M., Gazmararian, J., \& Nurss, J. (1999). Development of a brief test to measure functional health literacy. Patient Education and Counseling, 38(1), 33-42. http://doi.org/10.1016/S07383991(98)00116-5

Barsky, A. J., Saintfort, R., Rogers, M. P., \& Borus, J. F. (2002). Nonspecific medication side effects and the nocebo phenomenon. Jama, 287(5), 622. https://doi.org/10.1001/jama.287.5.622

Baugh, C. M., Robbins, C. a., Stern, R. a., \& McKee, A. C. (2014). Current understanding of chronic traumatic encephalopathy. Current Treatment Options in Neurology, 16(9). https://doi.org/10.1007/s11940-014-0306-5

Belanger, H. G., Curtiss, G., Demery, J., Lebowitz, B. K., \& Vanderploeg, R. D. (2005). Factors moderating neuropsychological outcomes following mild traumatic brain injury: a meta-analysis. Journal of the International Neuropsychological Society: JINS, 11(3), 215-227. http://doi.org/10.1017/S1355617705050277

Bell, T. R., \& Sanderson, J. (2016). A Hit on American Football: A Case Study of Bottom-up Framing Through Op-Ed Readers' Comments. International Journal of Sport Communication, 9(4), 499-518.

Benjamini, Y. \& Hochberg, Y. (1995). Controlling the false discovery rate : a practical and powerful approach to multiple testing, Journal of the Royal Statistical Society, 57(1), 289-300.

Bigler, E. D. (2008). Neuropsychology and clinical neuroscience of persistent postconcussive syndrome. Journal of International Neuropsychological Society, 14(1), 1-22. http://doi.org/10.1017/s135561770808017x

Block, C., Fabrizio, K., Bagley, B., Hannah, J., Camp, S., Mindingall, N., ... Lokken, K. (2014). Assessment of veteran and caregiver knowledge about mild traumatic brain injury in a VA Medical Center. Journal of Head Trauma Rehabilitation, 29(1), 76-88. http://doi.org/10.1097/HTR.0b013e3182886d78

Block, C., West, S., \& Goldin, Y. (2016). Misconceptions and misattributions about traumatic brain injury: an integrated conceptual framework. Physical Medicine and Rehabillitation 8(1). http://doi.org/10.1016/j.pmrj.2015.05.022 
Bürkner, P. (2017) Bayesian Regression Models using Stan. R package version 1.7.0 http://mc-stan.org/

Bureau of Labor Statistics. (2014). American time use survey-2014 results [press release]. Retrieved from http://www.bls.gov/news.release/pdf/atus.pdf

Buschke, H., Kuslansky, G., Katz, M., Stewart, W. F., Sliwinski, M. J., Eckholdt, H. M., \& Lipton, R. B. (1999). Screening for dementia with the Memory Impairment Screen. Neurology, 52(2), 231-238.

Carter-Allison, S. N., Potter, S., \& Rimes, K. (2016). Diagnosis Threat and Injury Beliefs after Mild Traumatic Brain Injury. Archives of Clinical Neuropsychology, 31(7), 727-737. https://doi.org/10.1093/arclin/acw062

Carroll, L. J., Cassidy, J. D., Peloso, P. M., Borg, J., Von Holst, H., Holm, L., ... Pépin, M. (2004). Prognosis for mild traumatic brain injury: Results of the WHO Collaborating Centre Task Force on mild traumatic brain injury. Journal of Rehabilitation Medicine, 36(SUPPL. 43), 84-105. http://doi.org/10.1080/16501960410023859

Cassilo, D., \& Sanderson, J. (2016). "I Dont Think Its Worth the Risk": Media Framing of the Chris Borland Retirement in Digital and Print Media. Communication \& Sport, 1-25. https://doi.org/10.1177/2167479516654513

Cassidy, J. D., Boyle, E., \& Carroll, L. J. (2014). Population-based, inception cohort study of the incidence, course, and prognosis of mild traumatic brain injury after motor vehicle collisions. Archives of Physical Medicine and Rehabilitation, 95(3 Suppl), S278-85. http://doi.org/10.1016/j.apmr.2013.08.295

Cassidy, J. D., Carroll, L. J., Peloso, P. M., Borg, J., Von Holst, H., Holm, L., ... Coronado, V. G. (2004). Incidence, risk factors and prevention of mild traumatic brain injury: Results of the WHO Collaborating Centre Task Force on mild traumatic brain injury. Journal of Rehabilitation Medicine, 36(SUPPL. 43), 2860. http://doi.org/10.1080/16501960410023732

Cicerone, K. D., \& Azulay, J. (2002). Diagnostic utility of attention measures in postconcussion syndrome. The Clinical Neuropsychologist, 16(3), 280-289. http://doi.org/10.1076/clin.16.3.280.13849

Cissel, M. (2012). Media Framing: a comparative content analysis on mainstream and alternative new coverage of Occupy Wall Street. The Elon Journal of Undergraduate Research in Communications, 67-77. Retrieved from https://www.elon.edu/docs/eweb/academics/communications/research/vol3no1/08CisselEJSpring12.pdf 
Coleman, R., Thorson, E., \& Wilkins, L. (2011). Testing the effect of framing and sourcing in health news stories. J Health Commun, 16(9), 941-954. https://doi.org/10.1080/10810730.2011.561918

Crawford, J. R., Obonsawin, M. C., \& Allan, K. M. (1998). PASAT and Components of WAIS-R Performance: Convergent and Discriminant Validity. Neuropsychological Rehabilitation, 8(3), 255-272. https://doi.org/10.1080/713755575

De Vreese, C. H., \& Lecheler, S. (2012). What a Difference a Day Makes? The Effects of Repetitive and Competitive News Framing Over Time. Communication Research, 40(2), 147-175. https://doi.org/10.1177/0093650212470688

DiFazio, M., Silverberg, N. D., Kirkwood, M. W., Bernier, R., \& Iverson, G. L. (2015). Prolonged Activity Restriction After Concussion: Are We Worsening Outcomes? Clinical Pediatrics. http://doi.org/10.1177/0009922815589914

Faul, M., Xu, L., Wald, M. M., \& Coronado, V. (2010). Traumatic brain injury in the United States: emergency department visits, hospitalizations, and deaths. Centers for Disease Control and Prevention \& National Center for Injury Prevention and Control, 891-904. http://doi.org/10.1016/B978-0-444-52910-7.00011-8

Ferrell, R. B., \& Tanev, K. S. (2002). Traumatic brain injury in older adults. Current Psychiatry Reports, 4(5), 354-62. Retrieved from http://www.ncbi.nlm.nih.gov/pubmed/12230964

Finkelstein, E, Corso, P, Miller, T. (2006). The Incidence and Economic Burden of Injuries in the United States. New York: Oxford University Press.

Frencham, K. A., Fox, A. M., \& Maybery, M. T. (2005). Neuropsychological studies of mild traumatic brain injury: A meta-analytic review of research since 1995. Journal of Clinical and Experimental Neuropsychology, 27(3), 334-351.

Frost, R. B., Farrer, T. J., Primosch, M., \& Hedges, D. W. (2013). Prevalence of traumatic brain injury in the general adult population: A meta-analysis. Neuroepidemiology, 40(3), 154-159. http://doi.org/10.1159/000343275

Giza, C. C., \& Hovda, D. A. (2001). The Neurometabolic Cascade of Concussion. Journal of Athletic Training, 36(3), 228-235. http://doi.org/10.1227/NEU.0000000000000505

Goffman, E. (1974). Frame Analysis. New York: Harper \& Row.

Gronwall, D. (1977). Paced auditory serial-addition task: a measure of recovery from concussion. Perceptual and Motor Skills, 44, 367-373. 
Hoffman, S. J., \& Justicz, V. (2016). Automatically quantifying the scientific quality and sensationalism of news records mentioning pandemics: validating a maximum entropy machine-learning model. Journal of Clinical Epidemiology, 1-9. https://doi.org/10.1016/j.jclinepi.2015.12.010

Hou, R., Moss-Morris, R., Peveler, R., Mogg, K., Bradley, B. P., \& Belli, A. (2012). When a minor head injury results in enduring symptoms: a prospective investigation of risk factors for postconcussional syndrome after mild traumatic brain injury. https://doi.org/10.1136/jnnp-2011-300767

Institute of Medicine. (2004). Health Literacy: A Prescription to End Confusion. Board on Neuroscience and Behavioral Health, Committee on Health Literacy. Washington, DC.

Iverson, G. L. (2005). Outcome from mild traumatic brain injury. Current Opinion in Psychiatry, 18(3), 301-317. http://doi.org/10.1097/01.yco.0000165601.29047.ae

Iverson, G. L., Brooks, B. L., Ashton, V. L., \& Lange, R. T. (2010). Interview versus questionnaire symptom reporting in people with the postconcussion syndrome. The Journal of Head Trauma Rehabilitation, 25(1), 23-30. http://doi.org/10.1097/HTR.0b013e3181b4b6ab

Karantzoulis, S., \& Randolph, C. (2013). Modern chronic traumatic encephalopathy in retired athletes: What is the evidence? Neuropsychology Review, 23(4), 350-360. http://doi.org/10.1007/s11065-013-9243-4

Kennard, M., Mclellan, T., \& Mckinlay, A. (2017). Sports Media Representations of Concussions in the National Rugby League. Australian Psychologist, 1-6. https://doi.org/10.1111/ap.12272

King, N.S., Crawford, S., Wenden, F.J., Moss, N.E.G., Wade, D. T. (1995). The Rivermead Post Concussion Symptoms Questionnaire: a measure of symptoms commonly experienced after head injury and its reliability. Journal of Neurology, 242(9), 587-592.

Kit, K., Mateer, C., Tuokko, H., \& Spencer-Rodgers, J. (2014). Influence of negative stereotypes and beliefs on neuropsychological test performance in a traumatic brain injury population. Journal of the International Neuropsychological Society: JINS, 20(2), 157-67. http://doi.org/10.1017/S1355617713001264

Klemm, C., Das, E., \& Hartmann, T. (2014). Swine flu and hype: a systematic review of media dramatization of the H1N1 influenza pandemic. Journal of Risk Research, (September), 37-41. http://doi.org/10.1080/13669877.2014.923029 
Kumar, S., Rao, S. L., Chandramouli, B. A., \& Pillai, S. (2013). Reduced contribution of executive functions in impaired working memory performance in mild traumatic brain injury patients. Clinical Neurology and Neurosurgery, 115(8), 1326-1332. http://doi.org/10.1016/j.clineuro.2012.12.038

Kutner, M., Greenberg, E., Jin, Y., \& Paulsen, C. (2006). The Health Literacy of America's Adults. Institute of Education Sciences.

Laborey, M., Masson, F., Ribéreau-Gayon, R., Zongo, D., Salmi, L. R., \& Lagarde, E. (2014). Specificity of postconcussion symptoms at 3 months after mild traumatic brain injury: results from a comparative cohort study. The Journal of Head Trauma Rehabilitation, 29(1), E28-36. http://doi.org/10.1097/HTR.0b013e318280f896

Lamont, R., Swift, H. J., \& Abrams, D. (2015). A review and meta-analysis of age-based stereotype threat: negative stereotypes, not facts, do the damage. Psychology and Aging, 30(1), 180-193. http://doi.org/10.1037/a0038586

Langlois, J. a, Rutland-Brown, W., \& Wald, M. M. (2006). The epidemiology and impact of traumatic brain injury: a brief overview. The Journal of Head Trauma Rehabilitation, 21(5), 375-378. http://doi.org/00001199-200609000-00001 [pii]

Lannsjö, M., Geijerstam, J.-L. af, Johansson, U., Bring, J., \& Borg, J. (2009). Prevalence and structure of symptoms at 3 months after mild traumatic brain injury in a national cohort. Brain Injury. Retrieved from http://www.tandfonline.com/doi/full/10.1080/02699050902748356

Lipton, R. B., Katz, M. J., Kuslansky, G., Sliwinski, M. J., Stewart, W. F., Verghese, J., ... Buschke, H. (2003). Screening for dementia by telephone using the Memory Impairment Screen. Journal of the American Geriatrics Society, 51(10), 13821390. https://doi.org/10.1046/j.1532-5415.2003.51455.x

Maroon, J. C., Winkelman, R., Bost, J., Amos, A., Mathyssek, C., \& Miele, V. (2015). Chronic traumatic encephalopathy in contact sports: a systematic review of all reported pathological cases. Plos One, 10(2), e0117338. http://doi.org/10.1371/journal.pone.0117338

Matthews, G., Deary, I. J., Whiteman, M. C., \& Matthews, G. (2013). Baysian Data Analysis (3rd ed.). Boca Raton: Taylor \& Francis.

McCrea, M. (2008). Mild Traumatic Brain Injury and Postconcussion Syndrome: The New Evidence Base for Diagnosis and Treatment (1st editio). New York: Oxford University Press. 
Nestoriuc, Y., Orav, E. J., Liang, M. H., Horne, R., \& Barsky, A. J. (2010). Prediction of nonspecific side effects in rheumatoid arthritis patients by beliefs about medicines. Arthritis Care and Research, 62(6), 791-799. https://doi.org/10.1002/acr.20160

Nevelsteen, S., Legros, J., \& Crasson, M. (2007). Effects of information and $50 \mathrm{~Hz}$ magnetic fields on cognitive performance and reported symptoms.

Bioelectromagnetics, 28(1), 53-63. http://doi.org/10.1002/bem.20265

Pena, E. A., \& Slate, E. H. (2014). gvlma: Global validation of linear models assumptions. $R$ package version, $1(0.2)$.

Paniak, C., Reynolds, S., Phillips, K., Toller-Lobe, G., Melnyk, A., \& Nagy, J. (2002). Patient complaints within one month of mild traumatic brain injury: a controlled study. Archives of Clinical Neuropsychology: The Official Journal of the National Academy of Neuropsychologists, 17(4), 319-334.

http://doi.org/10.1016/S0887-6177(01)00115-9

R Core Team, (2015). R: A language and environment for statistical computing. R Foundation for Statistical Computing, Vienna, Austria. http://www.R-project.org/

R Core Team, (2017). R: A language and environment for statistical computing. Vienna, Austria. http://www.R-project.org/

Rao, S. M., Leo, G. J., Bernardin, L., \& Unverzagt, F. (1991). Cognitive dysfunction in multiple sclerosis: frequency, patterns, and prediction. Neurology, 41, 685-691.

Rao, S. M., Leo, G. J., Haughton, V. M., St. Aubin-Faubert, P., \& Bernardin, L. (1989). Correlation of magnetic resonance imaging with neuropsychological testing in multiple sclerosis. Neurology, 39, 161-166.

Reuben, A., Sampson, P., Harris, A. R., Williams, H., \& Yates, P. (2014). Postconcussion syndrome (PCS) in the emergency department: predicting and pre-empting persistent symptoms following a mild traumatic brain injury. Emergency Medicine Journal : EMJ, 31(1), 72-7. https://doi.org/10.1136/emermed-2012201667

Rohling, M. L., Binder, L. M., Demakis, G. J., Larrabee, G. J., Ploetz, D. M., \& Langhinrichsen-Rohling, J. (2011). A meta-analysis of neuropsychological outcome after mild traumatic brain injury: re-analyses and reconsiderations of Binder et al. (1997), Frencham et al. (2005), and Pertab et al. (2009). The Clinical Neuropsychologist, 25(4), 608-623. https://doi.org/dx.doi.org/10.1080/13854046.2011.565076 
Rohling, M. L., Larrabee, G. J., \& Millis, S. R. (2012). The "Miserable Minority" following mild traumatic brain injury: who are they and do meta-analyses hide them? The Clinical Neuropsychologist, 26(2), 197-213. http://doi.org/10.1080/13854046.2011.647085

Rosenthal, R. (1979). The file drawer problem and tolerance for null results. Psychological Bulletin, 86(3), 638-641. https://doi.org/10.1037/00332909.86.3.638

Roth, R. S., \& Spencer, R. J. (2013). Iatrogenic risk in the management of mild traumatic brain injury among combat veterans: a case illustration and commentary. International Journal of Physical Medicine \& Rehabilitation, 01(01), 1-7. http://doi.org/10.4172/2329-9096.1000105

Ruan, X., \& Kaye, A. D. (2015). Nocebo Effect of Informed Consent in Interventional Procedures. The Clinical Journal of Pain, 32(5), 460-462. https://doi.org/10.1097/AJP.0000000000000332

Schneiderman, A. I., Braver, E. R., \& Kang, H. K. (2008). Understanding sequelae of injury mechanisms and mild traumatic brain injury incurred during the conflicts in Iraq and Afghanistan: persistent postconcussive symptoms and posttraumatic stress disorder. American Journal of Epidemiology, 167(12), 1446-1452. https://doi.org/10.1093/aje/kwn068

Scheufele, D. (1999). Framing as a theory of media effects. Journal of Communication, 49(1), 103-122. https://doi.org/10.1111/j.1460-2466.1999.tb02784.x

Scheufele, D. A., \& Tewksbury, D. (2007). Framing, agenda setting, and priming: The evolution of three media effects models. Journal of communication, 57(1), 9-20.

Silverberg, N. D., \& Iverson, G. L. (2011). Etiology of the post-concussion syndrome: physiogenesis and psychogenesis revisited. Neurorehabilitation, 29(4), 317-329. http://doi.org/10.3233/NRE-2011-0708

Smith, D. H., Meaney, D. F., \& Shull, W. H. (2003). Diffuse axonal injury in head trauma. Journal of Head Trauma Rehabilitation, 18(4), 307-316. http://doi.org/00001199-200307000-00003 [pii]

Snell, D. L., Surgenor, L. J., Hay-Smith, E. J. C., Williman, J., \& Siegert, R. J. (2015). The contribution of psychological factors to recovery after mild traumatic brain injury: Is cluster analysis a useful approach? Brain Injury, 29(3), 291-299. http://doi.org/10.3109/02699052.2014.976594

Spencer, S. J., Logel, C., \& Davies, P. G. (2016). Stereotype Threat. Annual Review of Psychology, 67, 415-437. https://doi.org/10.1146/annurev-psych-073115-103235 
Stålnacke, B. M., Björnstig, U., Karlsson, K., \& Sojka, P. (2005). One-year follow-up of mild traumatic brain injury: Post-concussion symptoms, disabilities and life satisfaction in relation to serum levels of S-100B and neurone-specific enolase in acute phase. Journal of Rehabilitation Medicine, 37(5), 300-305.

https://doi.org/10.1080/16501970510032910

Stan Development Team (2017). RStan: The R interface to Stan. R package version 2.15.1http://mc-stan.org/

Steele, C. M. (1997). A threat in the air. How stereotypes shape intellectual identity and performance. The American Psychologist, 52(6), 613-629.

http://doi.org/10.1037/0003-066X.52.6.613

Strauss, E., Sherman, E., Spreen, O. (2006). A Compendium of Neuropsychological Tests: Administration, Norms, and Commentary (3rd ed.). New York: Oxford University Press.

Suhr, J. \& Gunstad, J. (2002). "Diagnosis Threat": the effect of negative expectations on cognitive performance in head injury. Journal of Clinical and Experimental Neuropsychology, 24(4), 448-457. http://doi.org/10.1076/jcen.24.4.448.1039

Suhr, J. \& Gunstad, J. (2005). Further exploration of the effect of "diagnosis threat" on cognitive performance in individuals with mild head injury. Journal of the International Neuropsychological Society: JINS, 11(1), 23-29. http://doi.org/10.1017/S1355617705050010

Torchiano, M. (2016). Efficient Effect Size Computation. R package version 0.7.1 http://github.com/mtorchiano/effsize/

Thompson, H. J., McCormick, W. C., \& Kagan, S. H. (2006). Traumatic brain injury in older adults: epidemiology, outcomes, and future implications. Journal of the American Geriatrics Society, 54(10), 1590-1595. http://doi.org/10.1111/j.15325415.2006.00894.x

Van Slooten, E., Friedman, D. B., \& Tanner, A. (2013). Are we getting the health information we need from the mass media? An assessment of consumers' perceptions of health and medical news. Journal of Consumer Health on the Internet, 17(1), 35-53. http://doi.org/10.1080/15398285.2013.756338

Vanderploeg, R. D., Belanger, H. G., \& Kaufmann, P. M. (2014). Nocebo effects and mild traumatic brain injury: legal implications. Psychological Injury and Law, 245-254. http://doi.org/10.1007/s12207-014-9201-3

Vanotti, S., Eizaguirre, M. B., Cores, E. V., Yastremis, C., Garcea, O., Salgado, P., \& Cáceres, F. (2016). Validation of the PASAT in Argentina. Applied Neuropsychology: Adult, 9095(March), 1-5. https://doi.org/10.1080/23279095.2015.1092150 
Waldron-Perrine, B., Tree, H. A., Spencer, R. J., Suhr, J., \& Bieliauskas, L. (2015). Informational literature influences symptom expression following mild head injury: An analog study. Brain Injury, 00(00), 1-5.

http://doi.org/10.3109/02699052.2015.1004742

Weathers, F. W., Litz, B. T., Keane, T. M., Palmieri, P. A., Marx, B. P., \& Schnurr, P. P. (2013). The PTSD Checklist for DSM-5 (PCL-5). Retrieved from www.ptsd.va.gov

Witthöft, M., \& Rubin, G. J. (2013). Are media warnings about the adverse health effects of modern life self-fulfilling? An experimental study on idiopathic environmental intolerance attributed to electromagnetic fields (IEI-EMF). Journal of Psychosomatic Research, 74(3), 206-212.

http://doi.org/10.1016/j.jpsychores.2012.12.002

Young, G. (2008). Psychological injury and law: assumptions and foundations, controversies and myths, needed directions. Psychological Injury and Law, 1(1), 11-19. http://doi.org/10.1007/s12207-008-9005-4 


\title{
APPENDIX A
}

\section{REVISED HOFFMAN MEASUREMENT}

\author{
Tool for Measuring Scientific Quality and Sensationalism of News ${ }^{1}$
}

Your task is to watch a number of video clips about concussion and answer a few questions about their scientific quality and sensationalism. Scientific quality is a measure of a video's reliability and credibility on a given topic. Sensationalism is a way of presenting videos to make them seem more interesting or extraordinary than they actually are. Please familiarize yourself with this questionnaire and watch each video clip at least twice. Then, use this questionnaire to rate the video's scientific quality and sensationalism using a scale from 1 to 5 . Six questions evaluate the scientific quality of the video clip, and six questions evaluate sensationalism. Your work is helping to support important research being conducted at the University of Colorado Colorado Springs (USA) to improve health news coverage. For questions, please contact the principal investigator Cara Bussell at UCCS_Study@yahoo.com.

VIDEO CIIP\#:

0. Preliminary Question to Confirm Eligibiuty of Video Cuip [Please select "Yes" or "No"]

Is this a video clip primarily focused on concussion?

Yes $\square$ No $\square$

If "yes", please answer all remaining questions. If "no", please stop here and proceed to the next video clip.

A1. Scientific Quality: Appucability [Please select " 1 ", " 2 ", “ 3 ", “4" or " 5 "]

Is it clear to whom the information in the video is applicable and how it affects them?

An example of a very applicable video that deserves a " 5 " is:

"New medication could improve treatment for children with type 1 diabetes"

\begin{tabular}{|c|c|c|c|c|}
\hline No & \multicolumn{2}{c|}{ Partially } & \multicolumn{2}{c|}{ Yes } \\
\hline potentially misleading & \multicolumn{2}{|c|}{ minor lack of clarity } & \multicolumn{3}{c|}{ minimal ambiguity } \\
\hline 1 & 2 & 3 & 4 & 5 \\
\hline
\end{tabular}

A2. Scientific Quality: Opinions versus Facts [Please select "1", "2", "3", "4" or "5"] Are facts clearly distinguished from opinions?

An example of a statement that distinguishes between opinions and facts and deserves a " 5 " is:

"Most climate experts believe humans are the cause of global warming; others disagree"

\begin{tabular}{|c|c|c|c|c|}
\hline \multicolumn{2}{|c|}{ No } & \multicolumn{2}{c|}{ Partially } & \multicolumn{2}{c|}{ Yes } \\
\hline potentially misleading & $\begin{array}{c}\text { statements are attributed to sources, } \\
\text { but the underlying evidence is } \\
\text { ambiguous }\end{array}$ & \multicolumn{2}{c|}{$\begin{array}{c}\text { the evidence underlying the main } \\
\text { points is clearly cited }\end{array}$} \\
\hline 1 & 2 & 3 & 4 & \multicolumn{2}{|c|}{5} \\
\hline
\end{tabular}

A3. SCIENTIFIC QualitY: Validity [Please select " 1 ", "2", " 3 ", " 4 " or " 5 "]

Is there an assessment of the validity or credibility of information that is reported in a clear way?

An example of a valid statement that deserves a " 5 " is:

"Study findings may not be reliable due to the small sample size."

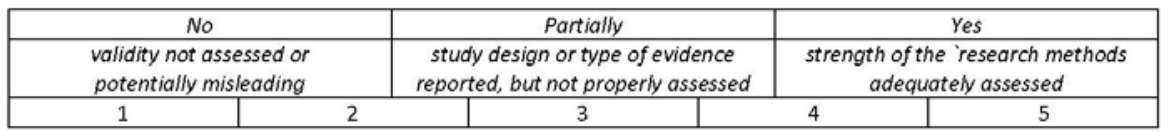

${ }^{1}$ Questions evaluating scientific quality were drawn from Oxman et al. 1993. Journal of Clinical Epidemiology 46(9):987-1001. Questions evaluating sensationalism were adapted from Molek-Kozakowska. 2013. Discourse \& Communication 7(2):173-197. Questionnaire was adapted from Hoffman, S. J., \& Justicz, V. 2016. Journal of Clinical Epidemiology, 1-9. 
A4. Scientific Quality: Precision [Please select " 1 ", " 2 ", " 3 ", " 4 " or " 5 "]

Is information reported in a precise way, such as about potential benefits, costs, risks of harm or trade-offs?

An example of a precise statement that deserves a " 5 " is:

"Seatbelts lower the risk of death and injury by $50 \%$ "

\begin{tabular}{|c|c|c|c|c|}
\hline \multicolumn{2}{|c|}{ No } & \multicolumn{2}{c|}{ Partially } & \multicolumn{2}{c|}{ Yes } \\
\hline not done or potentially misleading & \multicolumn{2}{c|}{$\begin{array}{c}\text { precision of effects or risks is } \\
\text { ambiguous }\end{array}$} & \multicolumn{2}{c|}{$\begin{array}{c}\text { precision of main effects or risks } \\
\text { clearly reported }\end{array}$} \\
\hline 1 & 2 & 3 & 4 & 5 \\
\hline
\end{tabular}

A5. Scientific Quality: Context [Please select " 1 ", " 2 ", " 3 ", "4" or "5"]

Is information reported in the broader context of what is scientifically known about the issue?

An example of a well-contextualized statement that deserves a " 5 " is:

"Several previous studies have confirmed that serotonin plays a role in many Autism cases"

\begin{tabular}{|c|c|c|}
\hline No & Partially & Yes \\
\hline not done or potentially misleading & $\begin{array}{l}\text { more than one study discussed but } \\
\text { some ambiguity on consistency }\end{array}$ & $\begin{array}{l}\text { mamy studies and consistency clearly } \\
\text { reported }\end{array}$ \\
\hline 1 & \begin{tabular}{l|l|l} 
& 3 & \\
\end{tabular} & \begin{tabular}{|l|l|}
4 & 5 \\
\end{tabular} \\
\hline
\end{tabular}

A6. Scientific Quality: Global Assessment [Please select " 1 ", " 2 ", " 3 ", "4" or " 5 "]

Based on your answers to the previous five questions, how would you rate the overall scientific quality of the video?

\begin{tabular}{|c|c|c|c|c|}
\hline \multicolumn{2}{|c|}{ Low } & \multicolumn{2}{c|}{ Moderate } & \multicolumn{2}{c|}{ migh } \\
\hline critical or extensive shortcomings & \multicolumn{1}{|c|}{$\begin{array}{c}\text { potentially important but not critical } \\
\text { shortcomings }\end{array}$} & \multicolumn{2}{c|}{ mings } \\
\hline 1 & 2 & 3 & 4 & 5 \\
\hline
\end{tabular}

B7. Sensationalism: Exposing [Please select " 1 ", " 2 ", " 3 ", " 4 " or " 5 "]

Does the video attempt to expose certain events, such as condemning a case of disease, failed policy, waste of money, or personal misbehaviour?

An example of an exposing statement that deserves a " 5 " is:

" $\$ 100$ to skip classes! Schools accused of bribing worst pupils to stay away when inspectors visit"

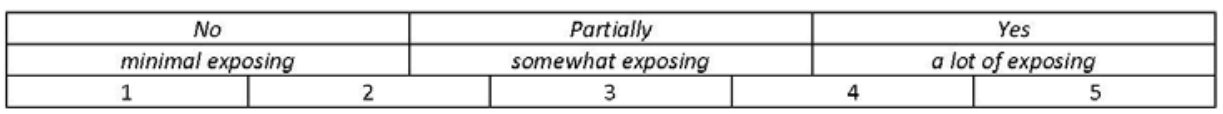

B8. Sensationalism: Speculating [Please select " 1 ", " 2 ", " 3 ", " 4 " or " 5 "]

Does the video offer a guess or suggest what the future consequences of an issue are likely to be?

An example of a speculative statement that deserves a " 5 " is:

"Hate preacher to go 'free in months': Radical cleric cannot be deported say European Human Rights judges"

\begin{tabular}{|c|c|c|c|c|}
\hline No & \multicolumn{2}{c|}{ Partially } & \multicolumn{2}{c|}{ Yes } \\
\hline minimal speculation & \multicolumn{3}{|c|}{ some speculation of speculation } \\
\hline 1 & 2 & 3 & 4 & 5 \\
\hline
\end{tabular}

B9. Sensationalism: Generalizing [Please select " 1 ", " 2 ", " 3 ", " 4 " or " 5 "]

Does the video make generalizing statements that extrapolate a trend out of an incident or pass a judgement about a whole class of people? An example of a generalizing statement that deserves a " 5 " is:

"Rise of the hugger mugger: 'Sociable' thieves who cuddle while they rob"

\begin{tabular}{|c|c|c|c|c|}
\hline No & \multicolumn{2}{c|}{ Partially } & \multicolumn{2}{c|}{ Yes } \\
\hline minimal generalizing & somewhat generalizing & \multicolumn{2}{c|}{ a lot of genering } \\
\hline 1 & 2 & 3 & 4 & 5 \\
\hline
\end{tabular}


B10. Sensationalism: Warning [Please select "1", "2", "3", “4" or " 5 "]

Does the video generate anxiety about an issue or offer suggestions on how to avoid becoming a victim?

An example of a statement that includes a strong warning and deserves a " 5 " is:

"A sausage a day could lead to cancer: Pancreatic cancer warning over processed meat"

\begin{tabular}{|l|c|c|c|c|}
\hline No & \multicolumn{2}{c|}{ Partially } & \multicolumn{2}{c|}{ Yes } \\
\hline minimal warnings & \multicolumn{3}{c|}{ some warnings of warnings } \\
\hline 1 & 2 & 3 & 4 & 5 \\
\hline
\end{tabular}

B11. Sensationalism: Extolung [Please select "1", "2", "3", "4" or "5"]

Does the video exaggerate facts as extraordinary, project events as historic, praise individuals for heroic acts, etc.?

An example of an extolling statement that deserves a " 5 " is:

"Teen victim heroically moves Prime Minister to bring in new drug-driving laws fit for a new century"

\begin{tabular}{|l|c|c|c|c|}
\hline \multicolumn{2}{|c|}{ No } & \multicolumn{2}{c|}{ Partially } & \multicolumn{2}{c|}{ Yes } \\
\hline minimal extolling & \multicolumn{2}{c|}{ somewhat extolling } & \multicolumn{2}{c|}{ a lot of extolling } \\
\hline 1 & 2 & 3 & 4 & 5 \\
\hline
\end{tabular}

B12. Sensationalism: Global Assessment [Please select " 1 ", " 2 ", " 3 ", " 4 " or " 5 "]

Based on your answers to the previous five questions, how would you rate the overall sensationalism of the video?

\begin{tabular}{|c|c|c|c|c|}
\hline \multicolumn{2}{|c|}{ Low } & Moderate & \multicolumn{2}{|r|}{ High } \\
\hline $\begin{array}{c}\text { not at all } \\
\text { sensationalizing }\end{array}$ & $\begin{array}{c}\text { not too much } \\
\text { sensationalizing }\end{array}$ & $\begin{array}{c}\text { somewhat } \\
\text { sensationalizing }\end{array}$ & $\begin{array}{c}\text { fairly } \\
\text { sensationalizing }\end{array}$ & very sensationalizing \\
\hline 1 & 2 & 3 & 4 & 5 \\
\hline
\end{tabular}

C13. Network[Please write in text]

Which Network displayed this video? 


\section{APPENDIX B \\ IRB APPROVAL

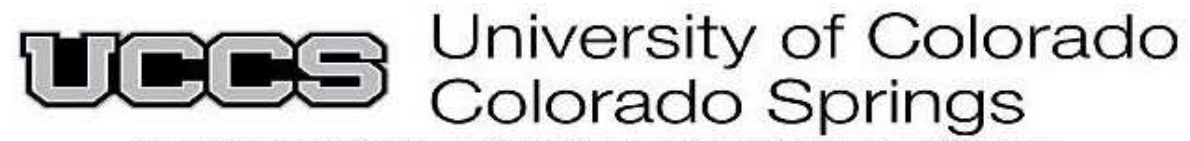 \\ Institutional Review Board (IRB) for the Protection of Human Subjects}

Date: $7 / 7 / 2016$

IRB Review

IRB PROT OCOL NO.: 16-241

Protocol Title: Media Sensationalization, Post Concussive Syndrome, and Cognitive Functioning APPROVED

Principal Investigator: Cara Bussell

Faculty Advisor if Applicable: Brandon Gavett

; Application: New Application

Type of Review: Exp edited 7

Risk L evel: No more than Minimal Risk

I Renewal Review Level (If changed from original approval) if Applic able: N/A No Change

1. This Protocol involves a Vulnerable Population: N/A (No Vulnerable Population)

Expires: 6 July 2017

*Note, if exempt: If there are no major changes in the research, protocol does not require review on a continuing basis by

i the IRB. In addition, the protocol may match more than one revi ew category not listed.

Externally funded: $\otimes N_{0} \square \mathrm{Yes}$

....... OSP \#: .... Sponsor:

Thank you for submitting your Request for IRB Review. The protocol identified above has been reviewed according to the policies of this institution and the provisions of applicable federal regulations. The review category is noted above, along with the expiration date, if applicable

Once human participant research has been approved, it is the Principal Investigator's (PI) responsibility to report any changes in research activity related to the project:

- The PI must provide the IRB with all protocol and consent form amendments and revisions.

- The IRB must approve these changes prior to implementation

- All advertisem ents recruiting study subjects must al so receive prior approval by the IRB

- The PI must promptly inform the IRB of all unanticipated serious adverse (within 24 hours). All unanticipated adver se events must be reported to the IRB within 1 week (see 45 CFR $46.103(b)($ ) $)$. Failure to comply with these feder ally mandated responsibilities may result in suspension or termination of the project.

- Renew study with the IRB prior to expriation

- Notify the IRB when the study is complete

If you have any questions, please contact Research Compliance Specialist in the Office of Sponsored Programs at 719-255-3903 or irbouccs.edu

Thank you for your concern about human subject protection issues, and good luck with your research.

Sincerely yours,

\section{zekCypress Vafkyrie}

Zek Cypress Valkyrie, PhD

IRB Reviewer 